\title{
THE MID-INFRARED EXTINCTION LAW IN THE OPHIUCHUS, PERSEUS, AND SERPENS MOLECULAR
} CLOUDS

\author{
Nicholas L. Chapman ${ }^{1,2}$, Lee G. Mundy ${ }^{1}$, Shih-Ping LaI ${ }^{3}$, And Neal J. Evans II ${ }^{4}$ \\ ${ }^{1}$ Department of Astronomy, University of Maryland, College Park, MD 20742, USA \\ 2 Jet Propulsion Laboratory, California Institute of Technology, 4800 Oak Grove Drive, MS 301-429, Pasadena, CA 91109, USA; Nicholas.L.Chapman@jpl.nasa.gov \\ ${ }^{3}$ Institute of Astronomy and Department of Physics, National Tsing Hua University, Hsinchu 30043, Taiwan \\ ${ }^{4}$ Department of Astronomy, University of Texas at Austin, 1 University Station C1400, Austin, TX 78712, USA \\ Received 2008 June 3; accepted 2008 September 3; published 2008 December 1
}

\begin{abstract}
We compute the mid-IR extinction law from 3.6 to $24 \mu \mathrm{m}$ in three molecular clouds-Ophiuchus, Perseus, and Serpens-by combining data from the "Cores to Disks" Spitzer Legacy Science program with deep $J H K_{s}$ imaging. Using a new technique, we are able to calculate the line-of-sight (LOS) extinction law toward each background star in our fields. With these LOS measurements, we create, for the first time, maps of the $\chi^{2}$ deviation of the data from two extinction law models. Because our $\chi^{2}$ maps have the same spatial resolution as our extinction maps, we can directly observe the changing extinction law as a function of the total column density. In the Spitzer Infrared Array Camera (IRAC) bands, 3.6-8 $\mu \mathrm{m}$, we see evidence for grain growth. Below $A_{K_{s}}=0.5$, our extinction law is well fitted by the Weingartner and Draine $R_{V}=3.1$ diffuse interstellarmedium dust model. As the extinction increases, our law gradually flattens, and for $A_{K_{s}} \geqslant 1$, the data are more consistent with the Weingartner and Draine $R_{V}=5.5$ model that uses larger maximum dust grain sizes. At $24 \mu \mathrm{m}$, our extinction law is $2-4$ times higher than the values predicted by theoretical dust models, but is more consistent with the observational results of Flaherty et al. Finally, from our $\chi^{2}$ maps we identify a region in Perseus where the IRAC extinction law is anomalously high considering its column density. A steeper near-IR extinction law than the one we have assumed may partially explain the IRAC extinction law in this region.
\end{abstract}

Key words: infrared: stars - ISM: clouds - stars: formation

Online-only material: color figures

\section{INTRODUCTION}

Dust is a ubiquitous component of the interstellar medium (ISM). It is important to understand the dust because it attenuates, or extincts, the light from background objects seen through the dust. The amount of extinction varies with both wavelength and position. Because of the constancy of the gas-to-dust ratio, the quantity of dust gives you a direct measure of the cloud's mass (Bohlin et al. 1978). Note, however, that the Bohlin et al. (1978) equation does depend on the $E(B-V)$ color excess, which can potentially vary depending on ISM properties such as metallicity. The dust extinction must also be accounted for when computing the luminosities for protostars within molecular clouds. The wavelength dependence of the extinction, known as the extinction law, directly relates to dust-grain properties such as size, composition, and the presence or lack of icy mantles on the grains.

Many authors have found that the near-IR (NIR) extinction law has the form of a power law, $A_{\lambda} \propto \lambda^{-\beta}$, between $\lambda \sim 1 \mu \mathrm{m}$ and $\sim 4 \mu \mathrm{m}$ with $\beta=1.6-1.8$ (Draine 2003, and references therein). The extinction law in the mid-IR wavelengths has not been as well studied. Rieke \& Lebofsky (1985) found the NIR power law extended out to $\sim 7 \mu \mathrm{m}$ before the extinctions increased again toward the $10 \mu \mathrm{m}$ silicate absorption peak. More recent results have found a different behavior. Lutz (1999) used Infrared Space Observatory to measure hydrogen recombination lines toward the Galactic center, and Indebetouw et al. (2005) and Flaherty et al. (2007) used the Spitzer Space Telescope to measure the mid-IR extinction law in several regions. These authors find a similar extinction law in the mid-IR which is much flatter than the Rieke \& Lebofsky (1985) result.
To date, no one has studied the changes in the extinction law within a large region with high spatial resolution. In this paper, we use Spitzer and deep ground-based $J H K_{s}$ observations to probe changes in the dust properties within three molecular clouds: Ophiuchus, Perseus, and Serpens. We compare our results with different dust models to investigate the relationship between dust-grain properties and total extinction (column density). In Section 2 we describe our observations. Then, in Section 3 we discuss our data reduction method, including how we identified background stars and computed line-of-sight (LOS) extinctions. In Sections 4 and 5 we create extinction and $\chi^{2}$ maps and compare them to each other. We also compute the average extinction law from 3.6 to $24 \mu \mathrm{m}$ for different ranges of extinction. Finally, we summarize our results in Section 6.

\section{OBSERVATIONS}

We mapped regions within the Ophiuchus, Perseus, and Serpens molecular clouds in eight wave bands from 1.25 to $24 \mu \mathrm{m}$. Our Spitzer observations (3.6-24 $\mu \mathrm{m})$ were part of the Spitzer Legacy Science program "From Molecular Cores to PlanetForming Disks" (c2d; Evans et al. 2003). The Spitzer observations have been previously published for Ophiuchus (Padgett et al. 2007), Perseus (Jørgensen et al. 2006; Rebull et al. 2007), and Serpens (Harvey et al. 2006, 2007b). To complement these data, we took $J H K_{s}$ observations at Kitt Peak National Observatory in three observing runs from 2004 September to 2006 June. We used the FLoridA Multi-object Imaging Near-IR Grism Observations Spectrometer (FLAMINGOS) (Elston 1998) on the 4-m telescope.

The c2d observations of Ophiuchus, Perseus, and Serpens cover approximately $11 \mathrm{deg}^{2}$ with both Spitzer's Infrared Array 


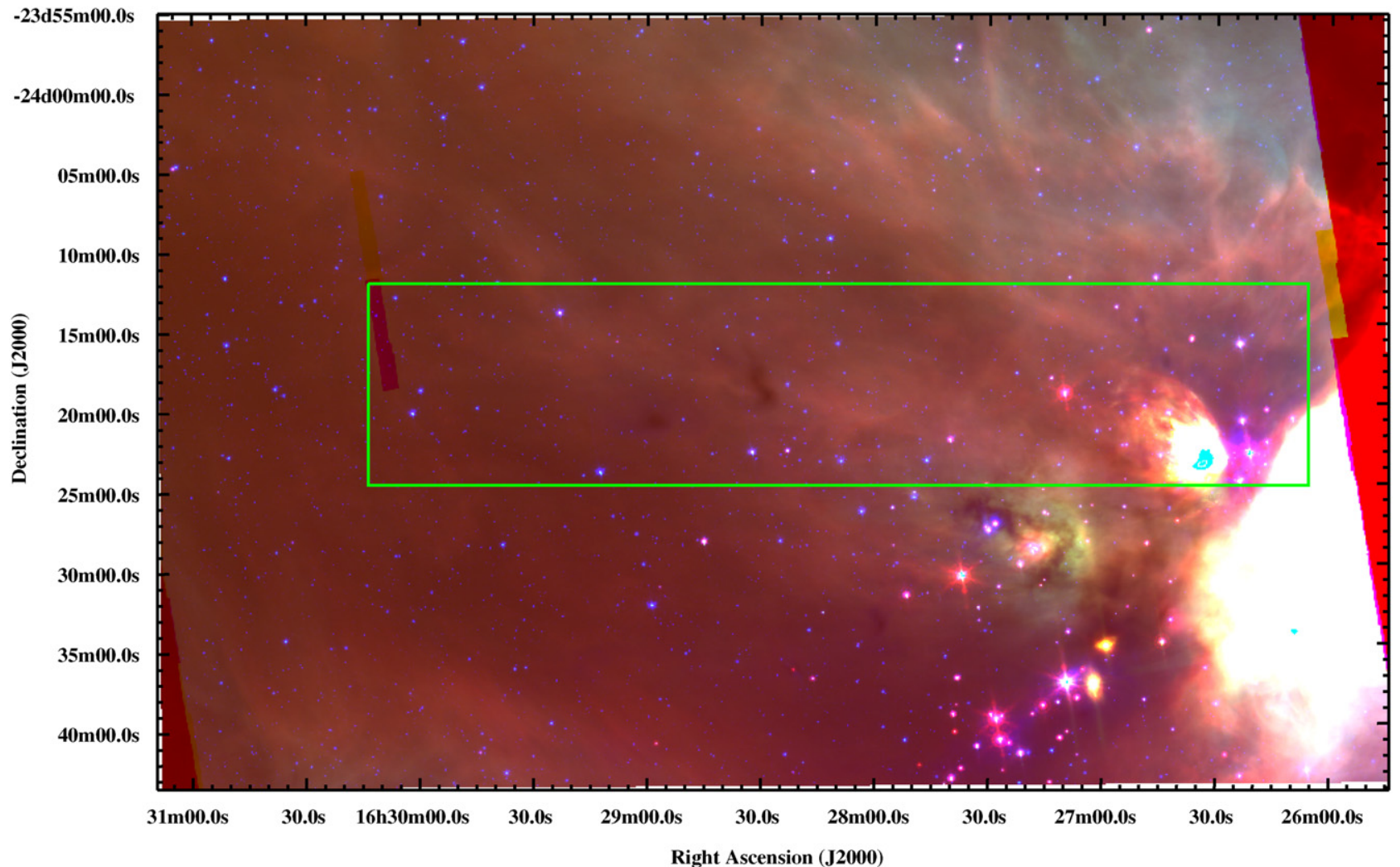

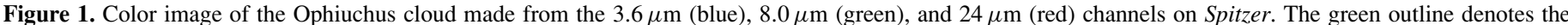
region we observed with our $J H K_{s}$ observations.

(A color version of this figure is available in the online journal.)

Table 1

Basic Properties of the Clouds

\begin{tabular}{lcrcc}
\hline \hline Cloud & $l$ & $b$ & $\begin{array}{c}\text { Dist. } \\
(\mathrm{pc})\end{array}$ & $\begin{array}{c}\text { Area Mapped in } J H K_{s} \\
\left(\mathrm{deg}^{2}\right)\end{array}$ \\
\hline Ophiuchus & 353 & 16 & $125 \pm 25^{\mathrm{a}}$ & 0.15 \\
Perseus & 160 & -19 & $250 \pm 50^{\mathrm{b}}$ & 1.0 \\
Serpens & 30 & 5 & $260 \pm 10^{\mathrm{c}}$ & 0.33 \\
\hline
\end{tabular}

References.

${ }^{a}$ de Geus et al. (1989).

${ }^{\mathrm{b}}$ Enoch et al. (2006).

c Straizys et al. (1996).

Camera (IRAC; 3.6-8 $\mu \mathrm{m}$ ) and Multiband Imaging Photometer for Spitzer (MIPS; $24 \mu \mathrm{m}$ ) instruments. Such a large area could not feasibly be observed by FLAMINGOS. Instead, we focused on selected regions within these clouds. Our goal was to explore changes in the extinction law within molecular clouds. Therefore, we chose contiguous regions with a range of extinction values, from low to high. Furthermore, we tried to include some star-forming regions within our mapped areas since these regions can alter the dust properties as well. In Table 1 we list the basic properties of these clouds including the total area mapped with our $J H K_{s}$ observations.

In Figures 1-3 we plotted a three-color image of each region using the c2d data. The red, green, and blue emissions correspond to the 24,8 , and $3.6 \mu \mathrm{m}$ channels on Spitzer, respectively. The green outline denotes the area we mapped in the $J H K_{s}$ bands.

In Ophiuchus we mapped a $\sim 10^{\prime} \times 60^{\prime}$ region to the northeast of L1688. The western side of our map, near L1688, contains many young stellar objects (YSOs) and several mid-IR dark cores are seen in the middle and on the eastern side. The $24 \mu \mathrm{m}$ emission is particularly bright on the western half of our map causing a saturated region to appear cyan in our color image.

Our Perseus observations also have a significant amount of dust nebulosity. A particularly bright region near the center of our map contains the IRAS source $03382+3145$. To the southeast of this region is a cluster of bright-red sources. Based on these sources' red appearance in Figure 2, they likely have an IR excess, suggesting they are YSOs. These sources were not resolved by IRAS, but IRAS $03388+3139$, a known IR source is located at this position. This cluster is identified by Jørgensen et al. (2006). Several other isolated IR excess sources exist within the field. Just to the east of our observed region is the well-known star-forming region IC348.

Finally, in Serpens we mapped a region surrounding "Cluster B," a group of YSOs to the south of the Serpens core region and located approximately in the middle of the $\mathrm{c} 2 \mathrm{~d}$ area. This region contains numerous YSOs. Two notable dark patches show up in the mid-IR Spitzer data, one to the southeast of "Cluster B" and the other to the northeast. Just to the south of our $J H K_{s}$ maps is the Herbig Ae star VV Ser surrounded by nebulosity.

\section{DATA REDUCTION}

We reduced our $J H K_{s}$ data using PyRAF. Each FLAMINGOS field consisted of multiple dithers with small offsets around a central position. To reduce our data, we first subtracted a medianfiltered dark image from each dither. Next, we flat-fielded the dithers by dividing out a median-filtered flat dome. The last, and most important, step was to subtract out the sky. This is critical 


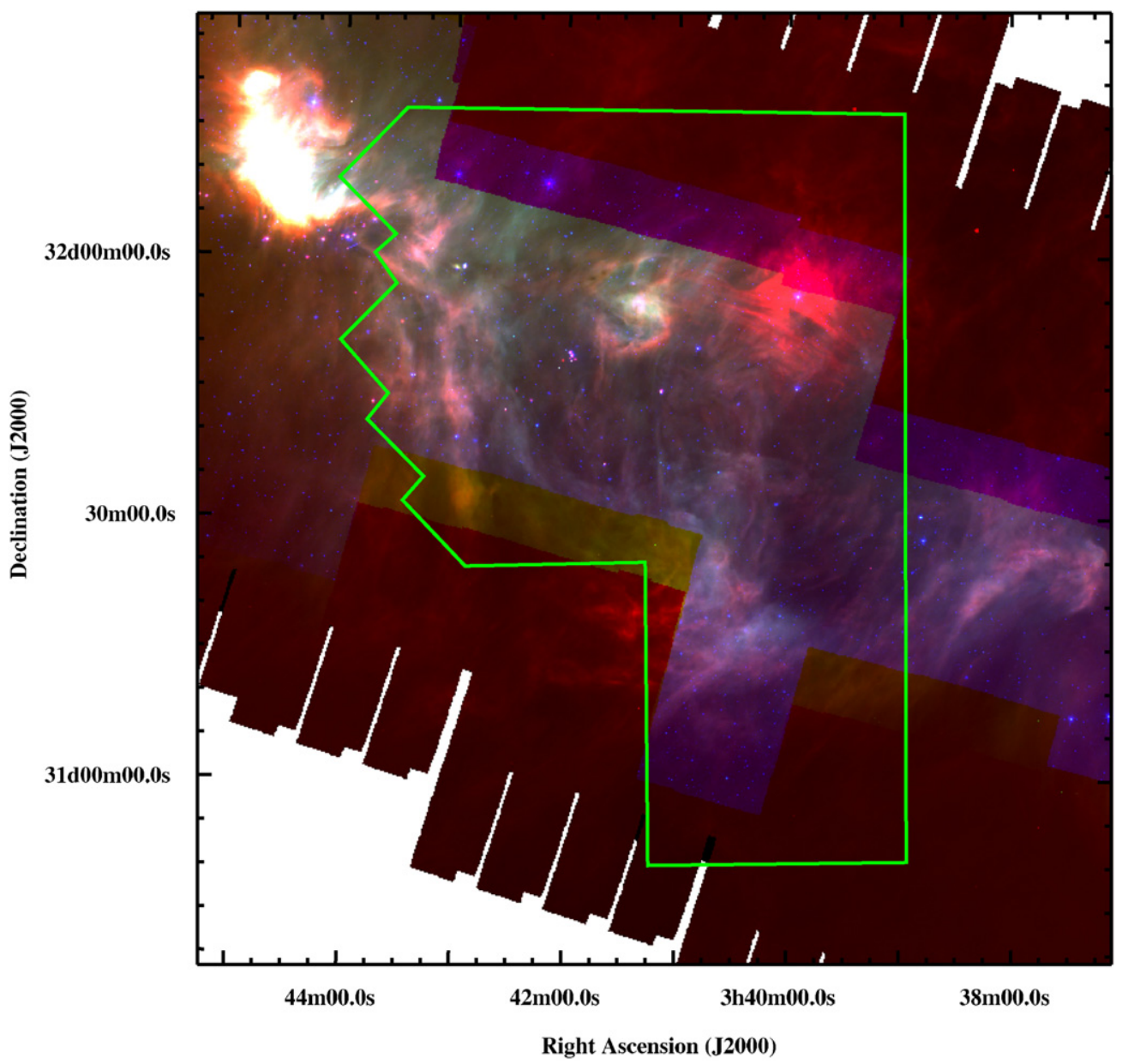

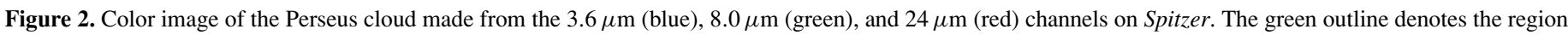
we observed with our $J H K_{s}$ observations.

(A color version of this figure is available in the online journal.)

for IR observations where the sky is typically much brighter than the astronomical objects of interest. We used a two-pass sky subtraction with a median sky computed from the nearest observed dithers in time. We identified the stars from the first pass, then masked them out in the second pass to produce an improved median-sky image.

The stars in each field were found using the IRAF task daofind, and daophot was used for the point spread function (PSF) photometry. We visually inspected the results to remove false sources found by daofind and also to add real sources missed by it. Finally, we used the Two Micron All Sky Survey (2MASS) catalog to correct the coordinates in each field and also to calibrate the photometry.

The Spitzer data were processed with the c2d data pipeline and are available on the Spitzer Science Center's (SSC's) Web site. ${ }^{5}$ Details on data reduction may be found in the $\mathrm{c} 2 \mathrm{~d}$ data-delivery documentation, also available on the Web site, and in several previously published papers (Padgett et al. 2007; Jørgensen et al. 2006; Rebull et al. 2007; Harvey et al. 2006; Harvey et al. 2007b).

\subsection{Data Quality}

The mean difference in position between sources in our $J H K_{s}$ catalogs and in the 2MASS catalogs is 0.15 and $95 \%$ of our

\footnotetext{
5 http://ssc.spitzer.caltech.edu/legacy/c2dhistory.html
}

sources have a difference in position of $\leqslant 0$.' 6 (around 2 pixels on the FLAMINGOS CCD). To test our photometric error, we computed the difference in flux between sources present in our Kitt Peak observations and the 2MASS catalogs. The resultant distribution is Gaussian with $\sigma=4-5 \%$.

The $\mathrm{c} 2 \mathrm{~d}$ delivery documentation discusses three sources of uncertainty: statistical, systematic, and absolute. The first two of these are incorporated into the photometric uncertainties listed in the $\mathrm{c} 2 \mathrm{~d}$ catalog. These errors are derived from the repeatability of flux measurements using the c2d pipeline. Since we are using the $\mathrm{c} 2 \mathrm{~d}$ data in this paper, we will adopt their uncertainties. The $\mathrm{c} 2 \mathrm{~d}$ documentation lists a $4.6 \%$ systematic error for IRAC and $9.2 \%$ for MIPS. Combining the systematic and statistical errors, our final photometric uncertainties are approximately $5 \%$ in IRAC and $10 \%$ in MIPS. The absolute uncertainties in the flux calibration are $1.5 \%$ and $4 \%$, respectively, for the IRAC and MIPS1 $(24 \mu \mathrm{m})$ bands. We obtained these values from the IRAC Data Handbook, version 3.0, and the MIPS Data Handbook, version 3.3.0. The absolute uncertainties are added in quadrature with the photometric errors during source classification, but are not listed in the final catalogs since they are smaller than the photometric errors.

Our $5 \sigma$ and $10 \sigma$ detection limits are listed in Table 2. For the $J H K_{s}$ bands our $10 \sigma$ limits are 19.5, 18.8, and $17.7 \mathrm{mag}$, respectively. These limits are $\sim 3.5$ mag deeper than $2 \mathrm{MASS}$ in all three bands. 


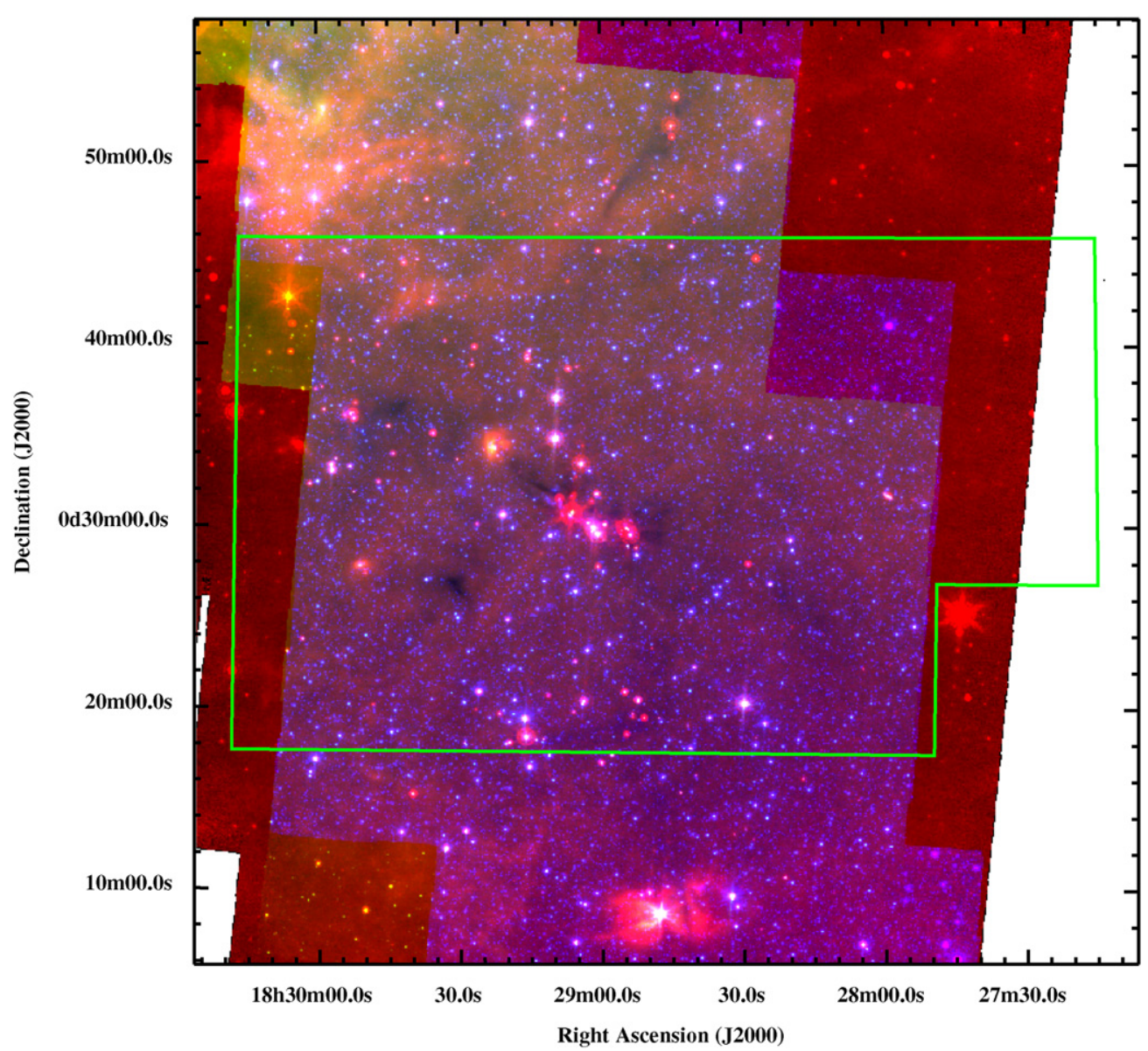

Figure 3. Color image of the Serpens cloud made from the $3.6 \mu \mathrm{m}$ (blue), $8.0 \mu \mathrm{m}$ (green), and $24 \mu \mathrm{m}$ (red) channels on Spitzer. The green outline denotes the region we observed with our $J H K_{s}$ observations.

(A color version of this figure is available in the online journal.)

Table 2

Magnitude Limits

\begin{tabular}{lcc}
\hline \hline Band & $10 \sigma$ Limits & $5 \sigma$ Limits \\
\hline$J$ & 19.5 & 20.3 \\
$H$ & 18.8 & 19.5 \\
$K_{s}$ & 17.7 & 18.4 \\
IRAC1 & 16.6 & 17.3 \\
IRAC2 & 15.9 & 16.6 \\
IRAC3 & 13.6 & 14.3 \\
IRAC4 & 12.8 & 13.5 \\
MIPS1 & 7.7 & 8.4 \\
\hline
\end{tabular}

\subsection{Extinction and Star Classification}

The standard c2d pipeline uses all available wavelengths to compute the extinction toward each star:

$$
\log \left(F_{\text {obs }}(\lambda) / F_{\text {model }}(\lambda)\right)=\log (k)-0.4 \times C_{\text {ext }}(\lambda) \times A_{V},
$$

where $F_{\text {model }}(\lambda)$ is the stellar photosphere model, $k$ is the scaling factor of the model for a particular star, and $C_{\text {ext }}(\lambda) \equiv A_{\lambda} / A_{V}$ is the ratio of extinction at wavelength $\lambda$ to visual extinction from the dust extinction law. The terms $k$ and $A_{V}$ are derived from the linear fit of this equation by adopting stellar photosphere and dust extinction models. The stellar photosphere models for $K_{s}$-MIPS1 bands are based on the Kurucz-Lejeune models and come from the SSC's "Star-Pet" tool. ${ }^{6}$ For the 2MASS

\footnotetext{
6 http://ssc.spitzer.caltech.edu/tools/starpet
}

bands, c2d translated the observed $J-H$ and $H-K$ colors of stars (Koornneef 1983) to fluxes relative to the $K$ band and ignored the difference between the $K$ and $K_{s}$ bands. Sources that were a good fit to this equation were classified as reddened stars; those that did not fit were compared with other templates to classify the sources.

In this paper, we slightly modified this procedure. First, the extinction, $A_{K_{s}}$, was computed for each source using the $J H K_{s}$ bands and the NICER (Near-Infrared Color Excess Revisited) technique (Lombardi \& Alves 2001). We also computed extinctions in $A_{K_{\mathrm{s}}}$ rather than the more traditional $A_{V}$ in order to directly compare our results with those of other authors. The NICER technique relies on the assumption of intrinsic values for the $J-H$ and $H-K_{s}$ colors of stars. For this paper we adopted the intrinsic colors $J-H=0.63 \pm 0.16$ and $H-K_{s}=0.21 \pm 0.14$. These values were based on the average color for stars from off-cloud fields. Then, using Equation (1), we identified the stars with the extinction held fixed.

We made this change because our primary goal in this paper is to compute the extinction law at the IRAC and MIPS wavelengths. The method we use for deriving the mid-IR extinction law $\left(A_{\lambda} / A_{K_{s}}\right)$ is dependent on $A_{K_{s}}$ so we cannot use these wavelengths when computing $A_{K_{s}}$. However, we still need to choose an appropriate extinction law. This will be used to compute $A_{K_{s}}$ from the $J H K_{s}$ bands and also for the source classification. For purposes of source classification, we considered two dust models, the Weingartner \& Draine (2001) $R_{V}=3.1$ and $R_{V}=5.5$ models. The $R_{V}=3.1$ model 


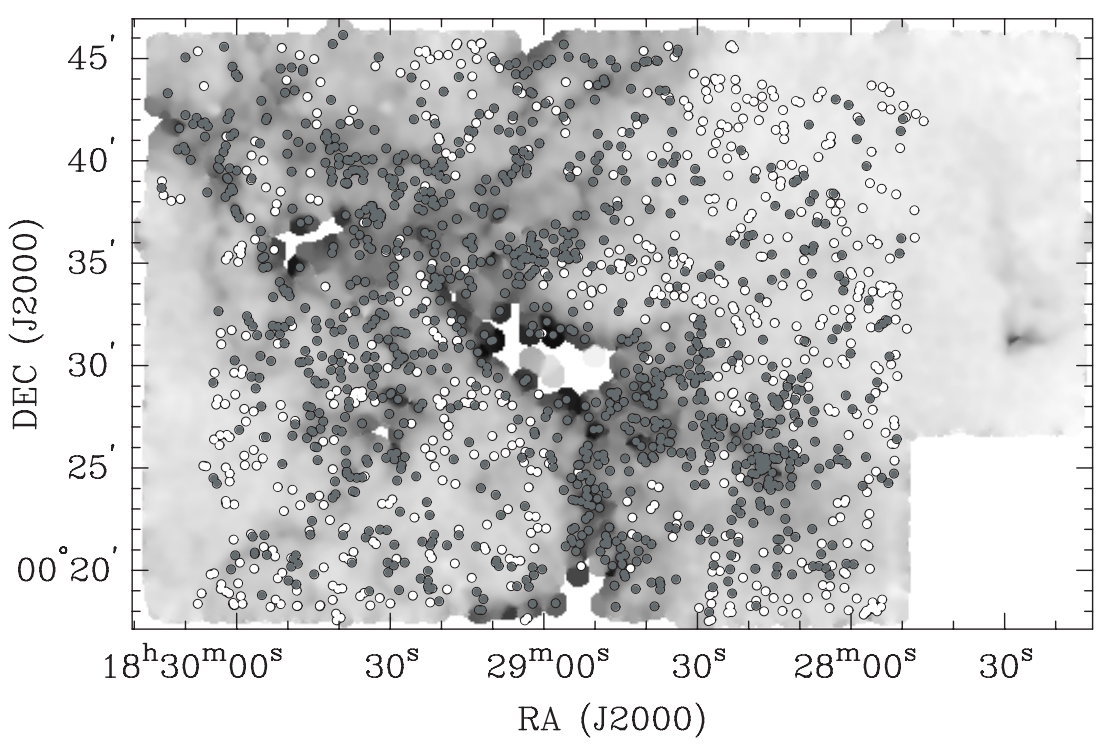

Figure 4. Extinction map of Serpens (see Section 4) with sources identified as stars using only the WD3.1 (white) or WD5.5 (dark gray) dust models.

(hereafter WD3.1) is designed to reproduce the extinction law of the diffuse ISM; the $R_{V}=5.5$ model (hereafter WD5.5) fits the observed law of denser regions. As we discuss in the following section, we adopted the WD5.5 dust model for computing the extinctions and source classifications. The parameter $R_{V}=A_{V} /\left(A_{B}-A_{V}\right)$ was historically used to characterize changes in the extinction law.

The advantage of using only the NIR bands to compute $A_{K_{s}}$ is that the extinction law in the NIR $J H K_{s}$ bands is very similar for both the WD3.1 and WD5.5 models. In Section 5.3 we will discuss possible biases in our results attributable to our assumption about the NIR extinction law.

\subsection{High-Reliability Star Catalogs}

We initially created high-reliability star catalogs by selecting only those sources classified as stars with both the WD3.1 and WD5.5 dust models. This selection excludes questionable stars that can affect our results, but may also miss bona fide stars that trace a true change in the extinction law. To check whether this happens, we plotted in Figure 4 those sources from Serpens classified as stars using either WD3.1 or WD5.5, but not both. We found that the sources classified as stars with WD5.5 are strongly associated with the higher extinction regions, while the WD3.1 stars are scattered randomly throughout the observed regions. Furthermore, up to a third of the detections that are only classified as stars using WD3.1 have a quality flag identifying them as confused with a nearby source suggesting that many of these sources have suspect photometry. In the remainder of the catalogs, less than $1 \%$ of the sources are similarly confused.

For these reasons, we selected all sources identified as stars with the WD5.5 dust model and computed LOS extinctions based on this model. We did not want a prominent absorption or emission line to bias our results. Therefore, we excluded any sources classified as stars only when one of the wave bands was dropped. Furthermore, because accurate extinction measurements are essential to this paper, we required sources to have a detection $\geqslant 7 \sigma$ in each of the $J H K_{s}$ bands. Finally, we "cleaned" our star catalogs to remove suspected faint background galaxies that were misidentified as stars. This procedure will be discussed in detail in Section 3.5. In our final catalogs we have the following number of sources before (after) cleaning: $2403(2365)$ in Ophiuchus, 11,479 $(11,280)$ in Perseus, and 49,712 $(49,485)$ in Serpens.

\subsection{Young Stellar Objects and Background Galaxies}

In addition to stars, the $\mathrm{c} 2 \mathrm{~d}$ classification procedure also identifies Young Stellar Object candidates (YSOc) and background galaxy candidates (Galc). Within the regions that we observed with deep $J H K_{s}$ and Spitzer observations, we identified the following number of YSOc (Galc) sources: 25 (6) in Ophiuchus, 40 (25) in Perseus, and 74 (21) in Serpens. Of course, many more YSOc and Galc objects are identified in the full-cloud catalogs. Readers interested in the YSO/Galc content of the full clouds should consult Padgett et al. (2007; Ophiuchus), Jørgensen et al. (2006) and Rebull et al. (2007; Perseus), and Harvey et al. (2007a; Serpens). All but 14 of our YSOc sources have the same identification in the c2d catalogs. Thirteen of the 14 are attributable to our modified source classification procedure. Our method tends to be conservative when classifying stars, thus ambiguous sources may become YSOs. In contrast, the c2d pipeline is more conservative when selecting YSOc sources, so ambiguous cases may become stars. The 14th source is classified as a YSOc in our catalogs, but as a Galc by c2d. This is because it is detected in our deep $J H K_{s}$ data but not by the 2MASS survey used by c2d. With our data, the c2d pipeline also classifies this source as a YSOc. In the c2d catalogs, this is source SSTc2d J034214.9+314758. All of our Galc sources are also identified as such by c2d.

\subsection{Misidentified Background Galaxies}

Figure 5 is a color-color diagram showing $J-H$ versus $H-K_{s}$ for our three clouds. Those sources brighter than the 15th magnitude at $K_{s}$ are shown in black, while fainter sources are gray crosses. We expect to see extincted stars following a reddening vector that stretches the data points into a line with some scatter, as shown in the figure. We do see this trend with the black points in all three clouds, and also with the gray crosses in Ophiuchus and Serpens. However, this trend is not clear in Perseus, where many gray sources with $H-K_{s} \gtrsim 0.7$ and $J-H \lesssim 1.4$ do not appear to follow the reddening vector.

What is the nature of these sources? One possibility is that these are merely faint stars with bad photometry. It is true that 


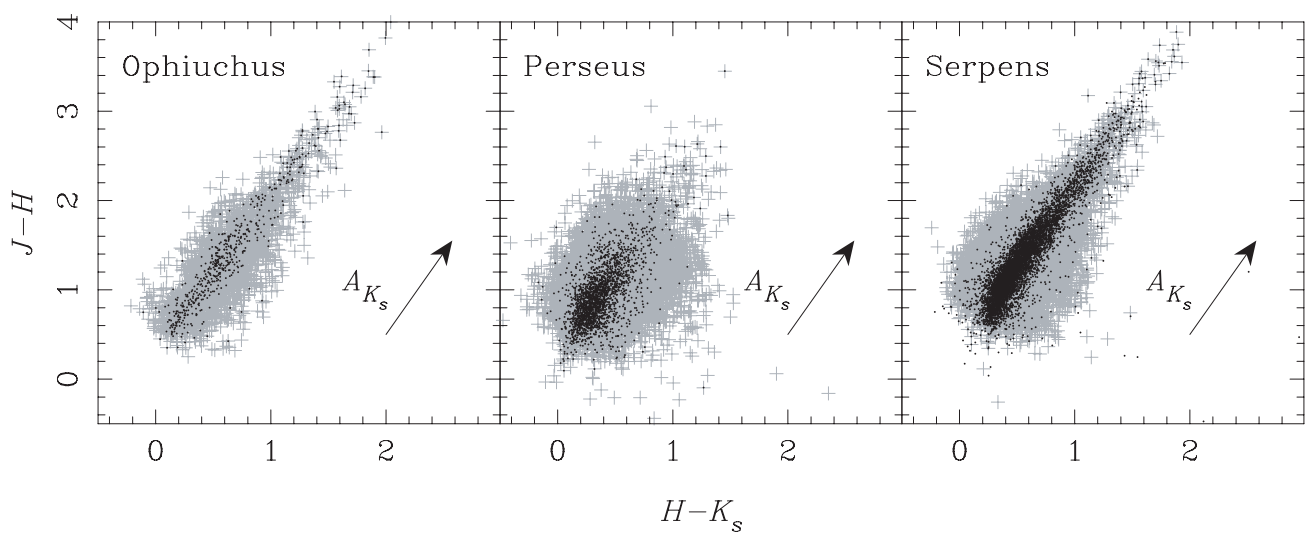

Figure 5. NIR color-color diagrams for Ophiuchus, Perseus, and Serpens. Gray crosses have $K_{s}$ magnitude greater than 15 , while the black points are brighter. The extinction vector shown is for the WD5.5 model.

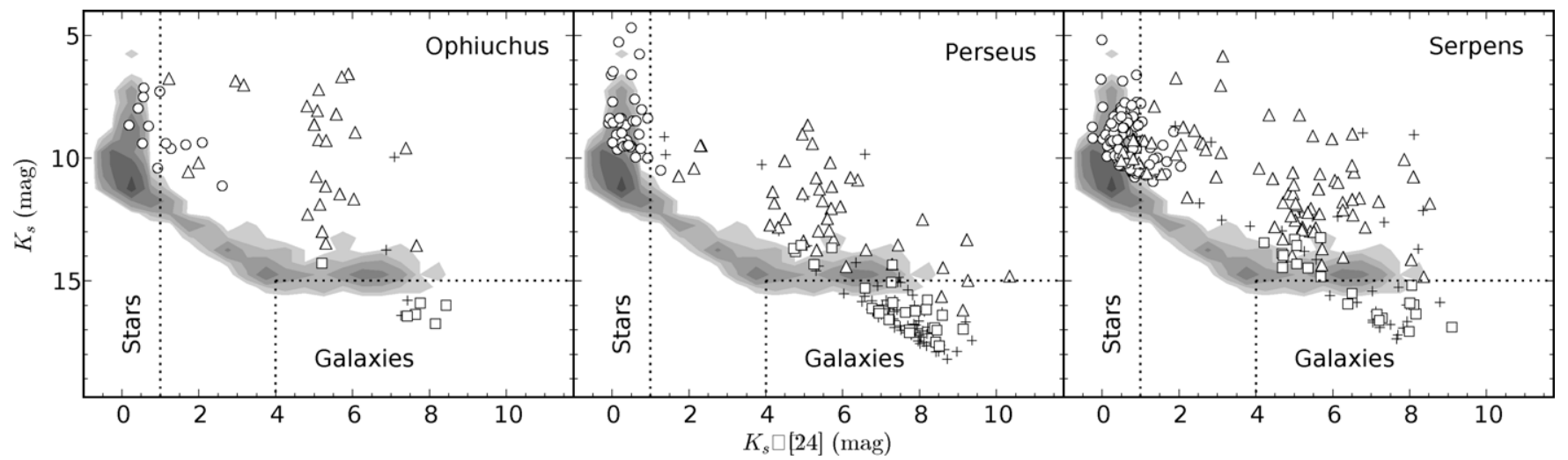

Figure 6. $K_{s}$ vs. $K_{s}-$ [24] plot for our three clouds. We used four different symbols to correspond to different source classifications: stars are circles, background galaxies are squares, "YSO candidates" are triangles, and plus signs denote all other classifications. The shaded contours are from the c2d processed catalog of part of ELAIS N1 (Surace et al. 2004). The dashed lines denote the cutoffs we used for selecting "known" stars and background galaxies.

these sources are fainter than the 15 th magnitude at $K_{s}$, but we are already using our high-reliability catalogs to ensure good photometry. Upon further examination, we found that many of these anomalous sources are only detected in the J-IRAC2 bands. Another possibility, therefore, is that these sources are not really stars even though they have been classified as such. Perseus is located at Galactic longitude $l=160^{\circ}$, almost in the Galactic anti-center direction, while Ophiuchus and Serpens are more toward the Galactic center at $l=353^{\circ}$ and $l=30^{\circ}$, respectively. With these Galactic longitudes and given that these anomalous sources are seen only in Perseus, we suspect these sources are likely misidentified background galaxies. Our hypothesis is supported by the recent work of Foster et al. (2008). They identified galaxies from near-infrared $J H K_{s}$ data and found that their galaxies were located in the same region of the $J-H$ versus $H-K_{s}$ color-color diagram as our suspected galaxies.

Our goal in this section is to develop a set of criteria that we will use to separate these suspected faint background galaxies from our actual stars. It is likely that any procedure will remove some bona fide stars and embedded objects in addition to background galaxies. We want our criteria to be stringent enough so that the final sample of sources selected for removal will be strongly enriched in background galaxies but also contain a minimum number of actual stars. We will then use this procedure to create purified star catalogs.

To determine whether the sources with anomalous colors really are background galaxies, we need a group of "known" stars and background galaxies with which we can compare. To make these "known" samples, we plotted $K_{s}$ versus $K_{s}-$ [24] for our three cloud regions in Figure 6 . We only plotted those sources with signal-to-noise ratio $(\mathrm{S} / \mathrm{N}) \geqslant 3 \sigma$ at $24 \mu \mathrm{m}$ in addition to the earlier cut $(7 \sigma)$ we made for uncertainties in $J H K_{s}$ photometry. The shaded contours in each panel are the Spitzer Wide-Area Infrared Extragalactic (SWIRE) survey data of European Large Area ISO Survey (ELAIS) N1 (Surace et al. 2004) as processed by the $\mathrm{c} 2 \mathrm{~d}$ team. The ELAIS N1 region is near the north Galactic pole, and therefore the data should contain nothing but stars and background galaxies, making them useful for selecting these type of objects. Based on Figure 6 we selected two groups of sources: those with $K_{s}-[24] \leqslant 1$ and those sources with $K_{s} \geqslant 15$ and $K_{s}-[24] \geqslant 4$. The first group, with no color excess, contains stars, while the second population consists of background galaxies. We will use these two groups as our "known" samples.

In Figure 7 we overlaid the "known" stars (white circles) and background galaxies (dark gray circles) on our $J-H$ versus $H-K_{s}$ color-color diagram. These two classes of sources are roughly separated. The stars chiefly lie along the stellar reddening vector, while the background galaxies appear below and to the right of this vector, exactly where our anomalous sources appear in Perseus. As our first selection criterion, we drew dashed lines to separate the stars from the possible galaxies. Note that the separation between the two is not perfect, but does establish that sources with $J-H \geqslant 0.6, H-K_{s} \geqslant 0.6$, and that to the right of the sloped line are more likely to be 


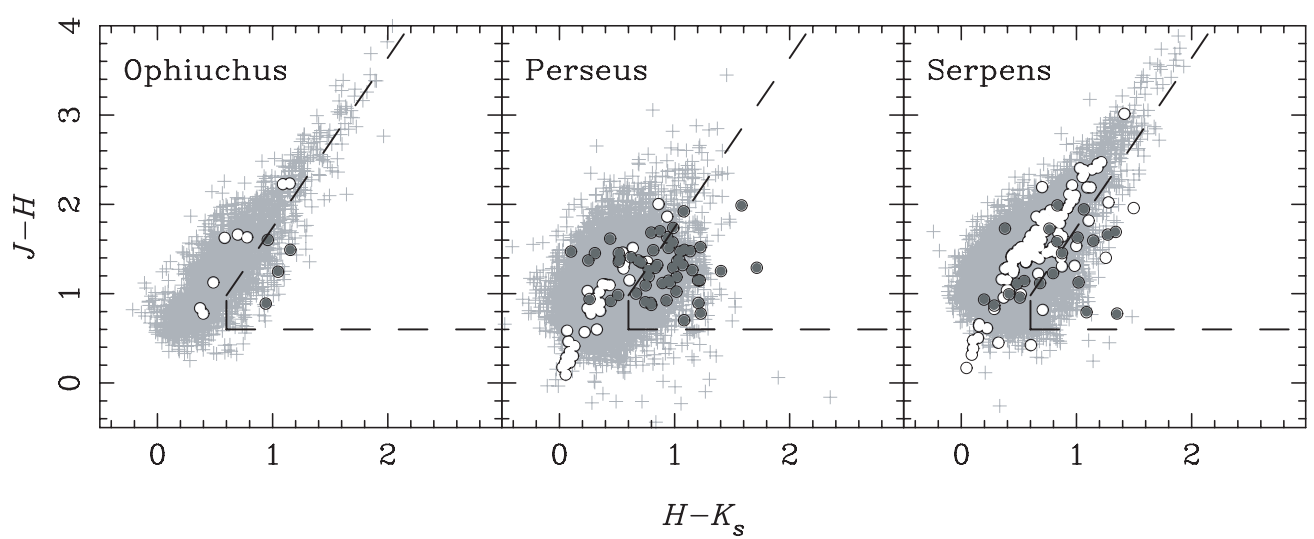

Figure 7. NIR color-color diagrams for Ophiuchus, Perseus, and Serpens. The white and dark gray circles are the "known" stars and background galaxies, respectively. The dashed lines select those sources with $J-H \geqslant 0.6, H-K_{s} \geqslant 0.6$, and $J-H \leqslant 1.9 \times\left(H-K_{s}\right)-0.16$.

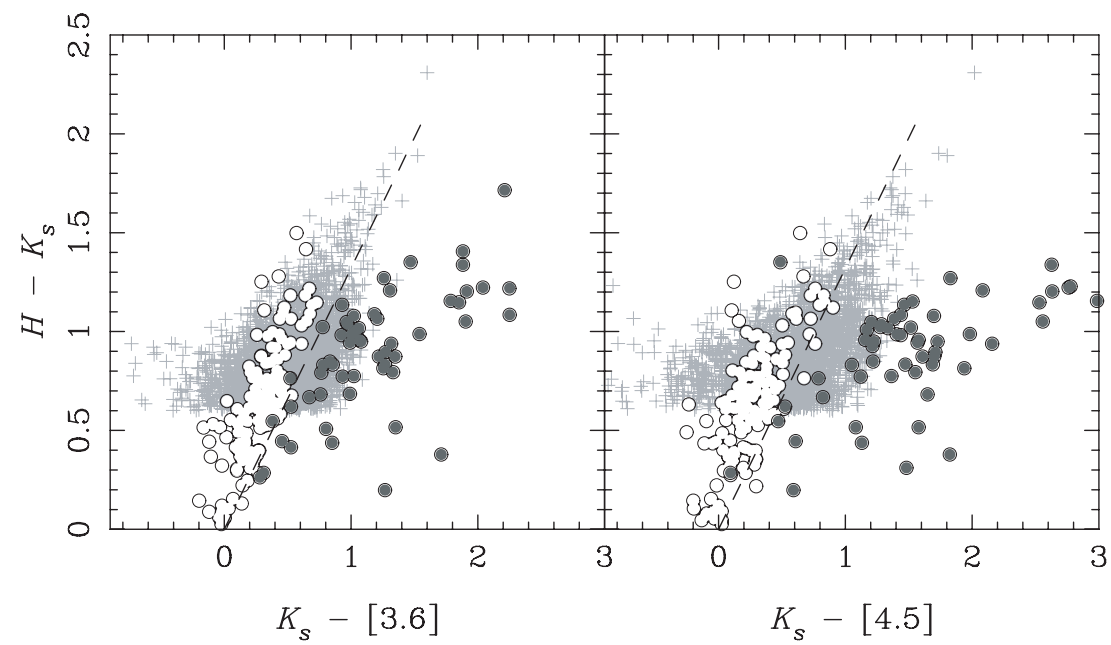

Figure 8. $H-K_{s}$ vs. $K_{s}-$ [3.6] (left) and vs. $K_{s}-$ [4.5] (right). The gray crosses are the sources selected by the dashed lines in Figure 7 for Ophiuchus, Perseus, and Serpens. The white and dark gray circles are the "known" stars and background galaxies, respectively. The dashed line in each panel has the equation $H-K_{s}=1.32 x$, where $x$ is either $K_{s}-[3.6]$ or $K_{s}-[4.5]$.

galaxies rather than stars. The equation for the sloped line is: $J-H=1.9 \times\left(H-K_{s}\right)-0.16$.

As noted earlier, approximately three-fourths of the objects in our clouds that lie in the region selected by our first criterion are faint enough that they are only detected in the $J$-IRAC2 wave bands. Therefore, in Figure 8 we plotted $H-K_{s}$ versus $K_{s}-$ [3.6] and versus $K_{s}-$ [4.5]. The gray crosses are those sources selected in all three clouds from Figure 7 by the dashed lines. Again, white circles are stars and dark gray circles are background galaxies. In both of these plots, the stars and galaxies are clearly separated. We defined a single line to use for both plots: $H-K_{s}=1.32 x$, where $x$ is either $K_{s}-$ [3.6] or $K_{s}-[4.5]$.

We combined these two selections to reduce the number of background galaxies in our data. Starting from the highreliability "star" catalogs, we removed all sources satisfying the following two criteria:

1. $J-H \geqslant 0.6, H-K_{s} \geqslant 0.6$, and $J-H \leqslant 1.9$ $\times\left(H-K_{s}\right)-0.16$.

2. $H-K_{s} \leqslant 1.32 \times\left(K_{s}-[3.6]\right)$ and $H-K_{s} \leqslant 1.32$ $\times\left(K_{s}-[4.5]\right)$.

Our cleaning procedure removed $1.6 \%$ of the sources in Ophiuchus, $1.8 \%$ in Perseus, and $0.5 \%$ in Serpens.

\section{EXTINCTION}

As mentioned in Section 3.2, we computed LOS extinctions, $A_{K_{s}}$, to each star using the NICER technique (Lombardi \& Alves 2001). These LOS $A_{K_{s}}$ values give us a randomly distributed sampling of the true $A_{K_{s}}$ in each cloud. To convert these LOS extinctions into a uniform map, we overlaid a grid on top of the data to represent the cells in the final extinction map. At each grid position, we used all of the LOS extinctions within a given radius. The extinction value in that cell is then the average of the individual extinctions, weighted both by uncertainty and distance from the center of the cell using a Gaussian weighting function.

For each cell we chose an integration radius equal to the full width at half maximum, or $2.3548 \sigma$. All that remains then is to choose the appropriate resolution for our maps. To obtain good statistical accuracy for each cell, we used a resolution that yielded on average 20+ stars in each cell. For Ophiuchus and Perseus, this corresponds to $90^{\prime \prime}$ resolution while in Serpens, which is toward the Galactic center, we were able to make a $30^{\prime \prime}$ resolution map and still have on average 36 stars per cell.

Foreground stars, which are correctly assigned a small $A_{K_{\mathrm{s}}}$, bias the average extinction for a given cell. We dealt with foreground stars as follows. For each cell with more than two stars, we computed the mean and median $A_{K_{s}}$ value. If these 


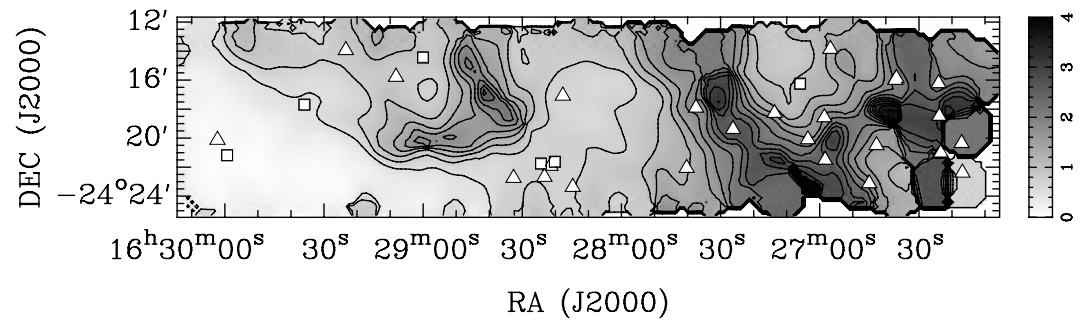

Figure 9. $A_{K_{s}}$ map of our observed region within the Ophiuchus cloud. The map has a resolution of $90^{\prime \prime}$. The contours start at $A_{K_{s}}=0.5$ in steps of $0.25(5 \sigma)$. We plotted all the YSOc and Galc objects as triangles and squares, respectively. The maximum $A_{K_{s}}$ value in the map is $4.0 \mathrm{mag}$.

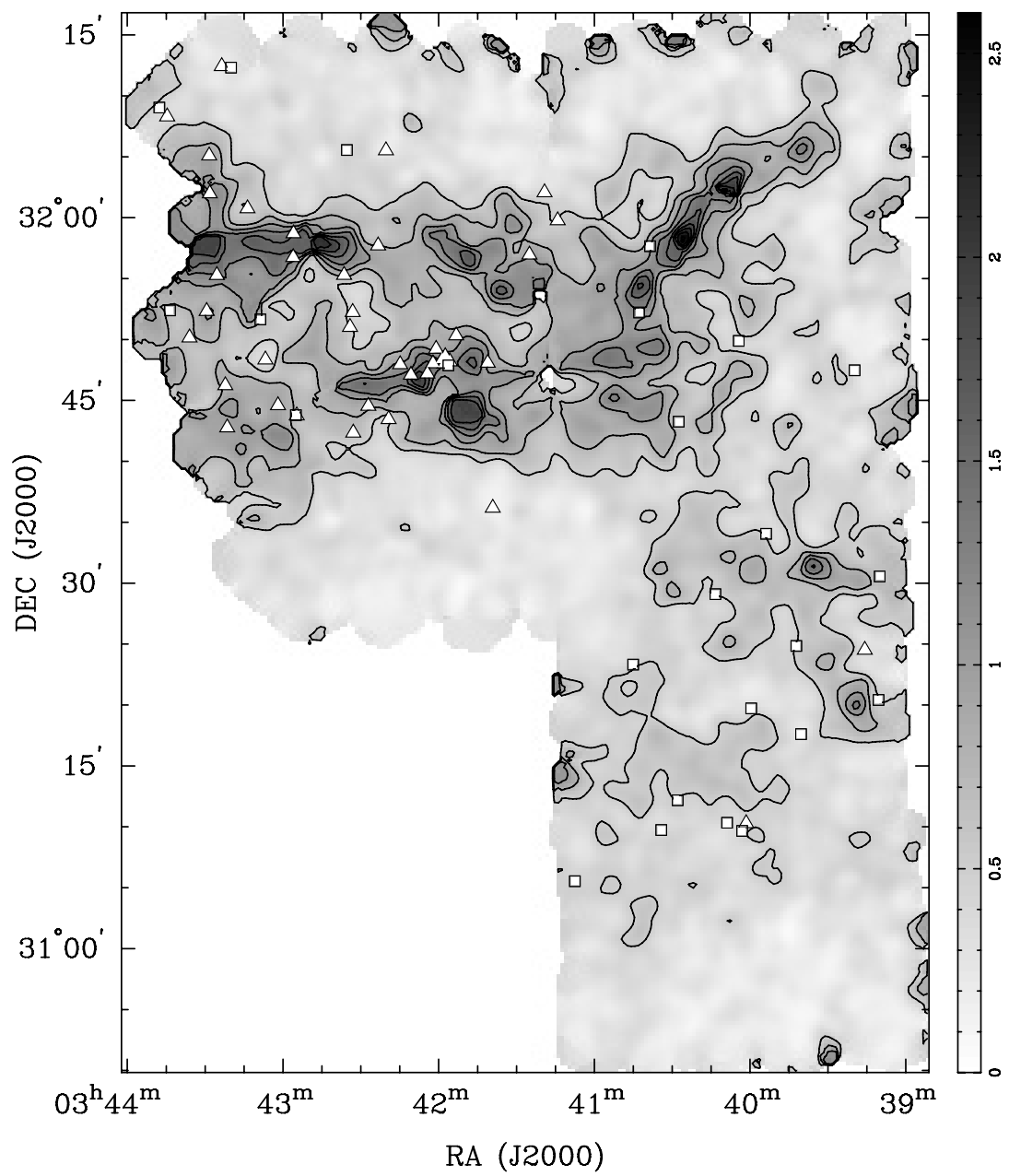

Figure 10. $A_{K_{S}}$ map of our observed region within the Perseus cloud. The map has a resolution of $90^{\prime \prime}$. The contours start at $A_{K_{s}}=0.5$ in steps of $0.25(5 \sigma)$. We plotted all the YSOc and Galc objects as triangles and squares, respectively. The maximum $A_{K_{s}}$ value in the map is $2.6 \mathrm{mag}$.

two statistics differed by more than $25 \%$, then we dropped the source with the lowest $A_{K_{s}}$ and recomputed the mean and median. However, if the difference between the old and new mean extinction is less than $25 \%$, then we re-added the dropped source to our catalog. After we identified all the sources to drop, we recomputed the extinctions in every cell. Our extinction maps of Ophiuchus, Perseus, and Serpens are shown in Figures 9-11.

Our Ophiuchus region has the largest dynamic range of $A_{K_{s}}$ in our clouds, up to $4 \mathrm{mag}$. A loop of extinction is located at $\sim 16^{\mathrm{h}} 28^{\mathrm{m}} 30^{\mathrm{s}}$ in addition to the high-extinction region L1688 on the western side of the map. Two-thirds of the YSOc sources are located in this half.

Our Perseus extinction map contains dust filaments in the upper half of the image and little extinction above the $A_{K_{s}}=0.5$ level elsewhere in the map. A cluster of YSOs identified as IRAS $03388+3139$ is located at R.A. $\sim 3^{\mathrm{h}} 42^{\mathrm{m}}$ decl. $\sim 31^{\circ} 47^{\prime}$. Furthermore, the high-extinction regions appear to be correlated with the darker patches in the color image. The dust lane that crosses the nebulous region of IRAS $03382+3145\left(\sim 3^{\mathrm{h}} 41^{\mathrm{m}} 30^{\mathrm{s}}\right.$ $+31^{\circ} 55^{\prime}$ ) shows up as a high-extinction filament in our extinction map. Almost all the Galc sources are in the low-extinction southern half of the map.

The Serpens extinction map has a higher resolution, $30^{\prime \prime}$, than either the Ophiuchus or Perseus maps. Two regions show up as holes in our maps because of a lack of background stars. The larger hole in the center of the map contains the star-forming region known as Cluster $\mathrm{B}$, while the extinction hole west of Cluster B shows up as a dark patch even at $24 \mu \mathrm{m}$. 


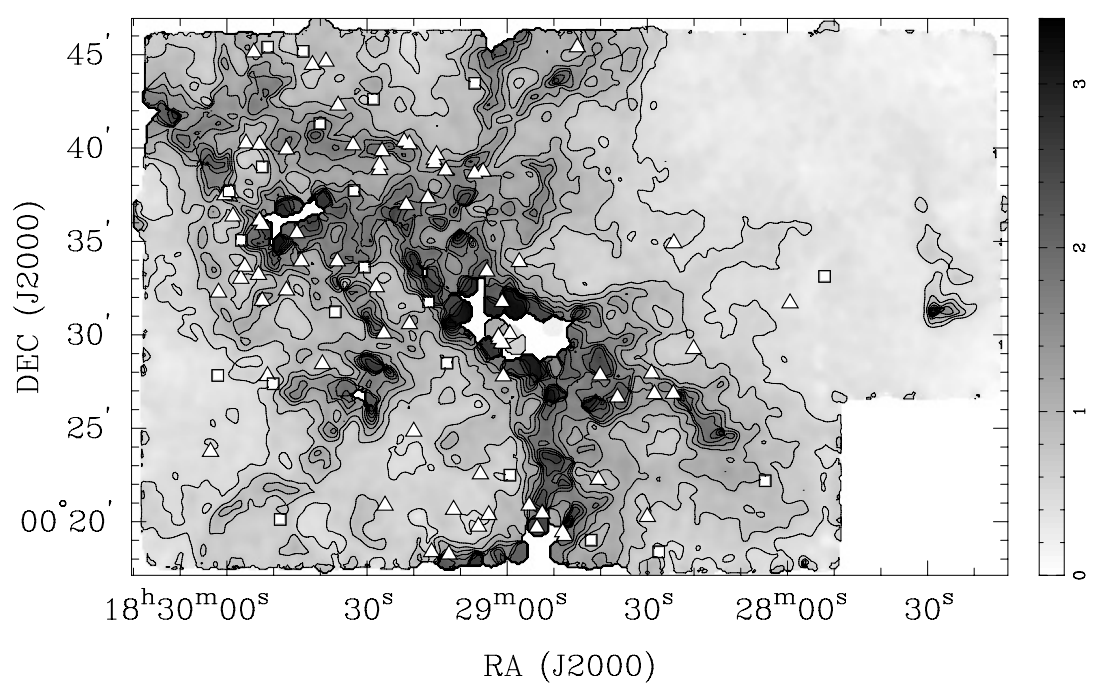

Figure 11. $A_{K_{s}}$ map of our observed region within the Serpens cloud. The "holes" in the map correspond to regions with no data either because they are too dense or because the only sources present were not classified as stars. The map has a resolution of $30^{\prime \prime}$. The contours start at $A_{K_{s}}=0.5$ in steps of $0.25(5 \sigma)$. We plotted all the YSOc and Galc objects for Serpens as triangles and squares, respectively. The maximum $A_{K_{s}}$ value in the map is $3.4 \mathrm{mag}$.

\section{DUST PROPERTIES}

The WD3.1 and WD5.5 models are nearly identical in the NIR $J H K_{s}$ bands. We will exploit this constancy of the extinction law in the NIR by using the extinctions computed from the $J H K_{s}$ bands and then extrapolating the extinction law to the Spitzer wave bands.

Starting from our basic equation relating flux and extinction,

$$
\log \left(F_{\text {obs }}(\lambda) / F_{\text {model }}(\lambda)\right)=\log (k)-0.4 \times C_{\text {ext }}(\lambda) \times A_{K_{s}},
$$

we can re-arrange this to solve for $C_{\text {ext }}$, defined as $A_{\lambda} / A_{K_{s}}$ :

$$
C_{\text {ext }}(\lambda)=\frac{2.5}{A_{K_{s}}}\left[\log (k)-\log \left(F_{\text {obs }}(\lambda) / F_{\text {model }}(\lambda)\right)\right] .
$$

Since we are only interested in differences in the extinction law, we subtracted $C_{\text {ext }}\left(K_{s}\right)$ to eliminate the need for $k$, the scaling factor. Furthermore, because $C_{\text {ext }}\left(K_{s}\right) \equiv 1$ and $F_{\text {model }}\left(K_{s}\right) \equiv 1$ (all stellar models are scaled relative to $K_{s}$ ), the equation simplifies to

$$
C_{\text {ext }}(\lambda)=\frac{2.5}{A_{K_{s}}}\left[\log \left(F_{\text {obs }}\left(K_{s}\right) / F_{\text {obs }}(\lambda)\right)+\log F_{\text {model }}(\lambda)\right]+1 .
$$

For $F_{\text {model }}(\lambda)$ we use the average stellar model derived in the Appendix.

\section{1. $\chi^{2}$ in the Clouds}

Our goal in this section is to construct two-dimensional maps of the changes in the dust properties across each cloud. We will do this by computing a reduced $\chi^{2}$ value along each LOS and then applying the same method we used to create $A_{K_{\mathrm{s}}}$ maps, except this time our "extinction" values will be $\chi^{2}$ values. We define $\chi^{2}$ as the sum of the difference between our computed extinction law and a theoretical model over the Spitzer IRAC wave bands $(3.6-8 \mu \mathrm{m})$. The reduced $\chi^{2}$ is then simply $\chi^{2}$ divided by the number of bands summed minus 1 , since we have one free parameter in the model, namely, $A_{K_{s}}$ :

$$
\chi^{2}=\frac{1}{n-1} \sum_{\lambda}^{n}\left(\frac{C_{\mathrm{ext}}^{\mathrm{obs}}(\lambda)-C_{\mathrm{ext}}^{\mathrm{model}}(\lambda)}{\sigma_{\lambda}}\right)^{2} .
$$

$C_{\mathrm{ext}}^{\mathrm{obs}}(\lambda)$ is computed from Equation (4), $C_{\mathrm{ext}}^{\mathrm{model}}$ is the extinction law for a given dust model, and $\sigma_{\lambda}$ is the uncertainty in $C_{\mathrm{ext}}^{\mathrm{obs}}$. We used both the WD3.1 and WD5.5 dust models in creating our $\chi^{2}$ maps. Note that we only summed over the IRAC bands. As we will see later, the extinction law at $24 \mu \mathrm{m}$ is almost always significantly higher than either the WD3.1 or WD5.5 extinction models (Section 5.2.2). We do not want to bias our results based on those data, so we excluded this data point when calculating the reduced $\chi^{2}$. Finally, we excluded any negative values for $C_{\mathrm{ext}}^{\mathrm{obs}}(\lambda)$ as unphysical.

We created maps of the $\chi^{2}$ values using the same process we used for extinction mapping and with the same resolution. Our $\chi^{2}$ maps for Ophiuchus, Perseus, and Serpens are shown in Figures 12-14. We drew our contours starting at $\chi^{2}=4$ because we observe a definite transition between the WD3.1 and WD5.5 dust models at this approximate $\chi^{2}$ value (see, Figure 15). Statistically, $\chi^{2}=4$ would arise by chance about $5 \%$ of the time for $n=2$.

In broad terms, high-extinction regions are correlated with high $R_{V}=3.1 \chi^{2}$ values. This same correlation is not seen with $R_{V}=5.5$. To make a quantitative comparison between $\chi^{2}$ and $A_{K_{\mathrm{s}}}$, we binned our data in $A_{K_{\mathrm{s}}}$ and for each bin determined the average $\chi^{2}$ with both the WD3.1 and WD5.5 models. Our results are shown in Figure 15. The $R_{V}=3.1 \chi^{2}$ curve is shown in black, while $R_{V}=5.5$ is shown in gray. At low extinction, $A_{K_{\mathrm{s}}} \lesssim 1$, the $R_{V}=3.1$ and 5.5 curves are very similar, suggesting it is difficult to distinguish between the dust models with our method. Above $A_{K_{s}} \approx 1$, the $R_{V}=3.1$ curve rises sharply, while $R_{V}=5.5$ does not.

Our increase in $R_{V}=3.1 \chi^{2}$ values in denser regions means the WD3.1 model does not fit the data in these regions. The WD5.5 model, which includes larger dust grains, produces much smaller $\chi^{2}$ values, suggesting grain growth is occurring in the denser regions. One prominent region within the Perseus $R_{V}=3.1 \chi^{2}$ map does not fit into this picture. This region is located at $\sim 3^{\mathrm{h}} 40^{\mathrm{m}} \cdot 5+31^{\circ} 30^{\prime}$ and circled in red in Figure 13. This region has a peak $\chi^{2}$ value of 39 , but since it is non-circular and larger than the integration radius, it seems unlikely that a single bad data point is affecting the $\chi^{2}$. The puzzling thing about this region is that the extinction is fairly 


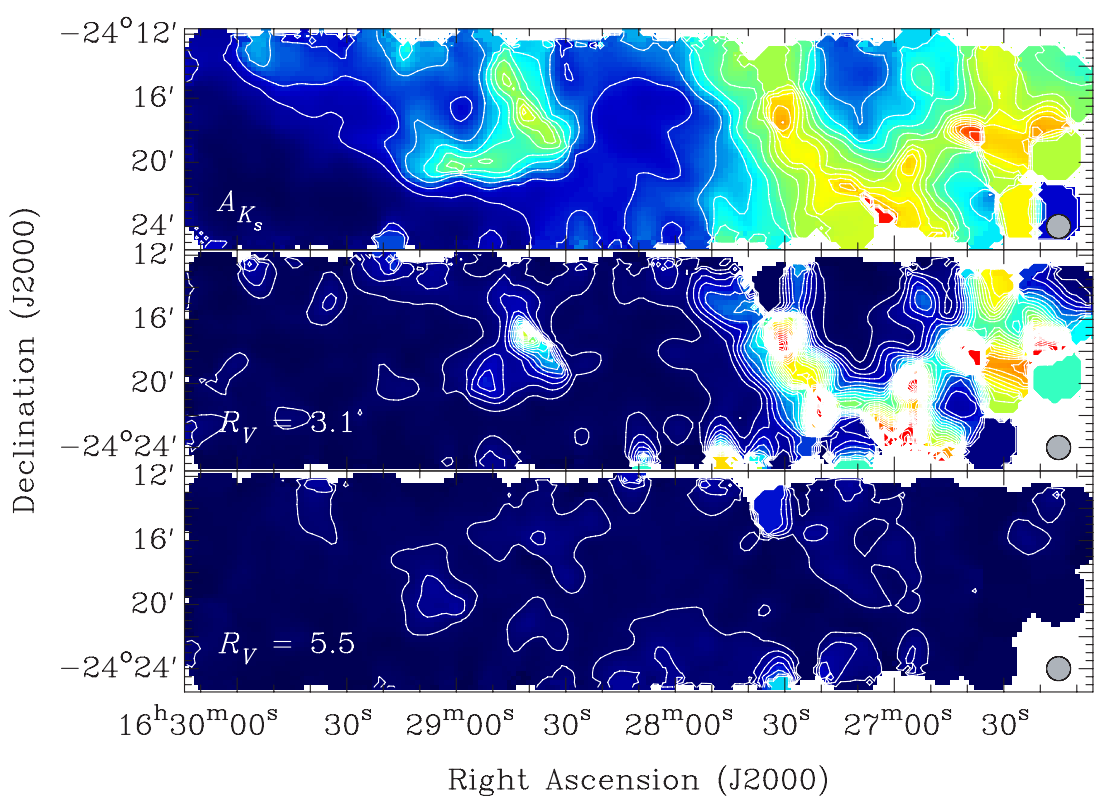

Figure 12. Map of the $A_{K_{s}}$ and $\chi^{2}$ values in Ophiuchus. The top panel shows the extinction map with contours starting at 0.5 mag in steps of 0.25 mag ( $5 \sigma$ ). The middle and bottom panels show the $\chi^{2}$ maps for the same region that were made assuming either the WD3.1 (middle) or WD5.5 (bottom) extinction laws. Contours start at $\chi^{2}=4$ in steps of 4 .

(A color version of this figure is available in the online journal.)

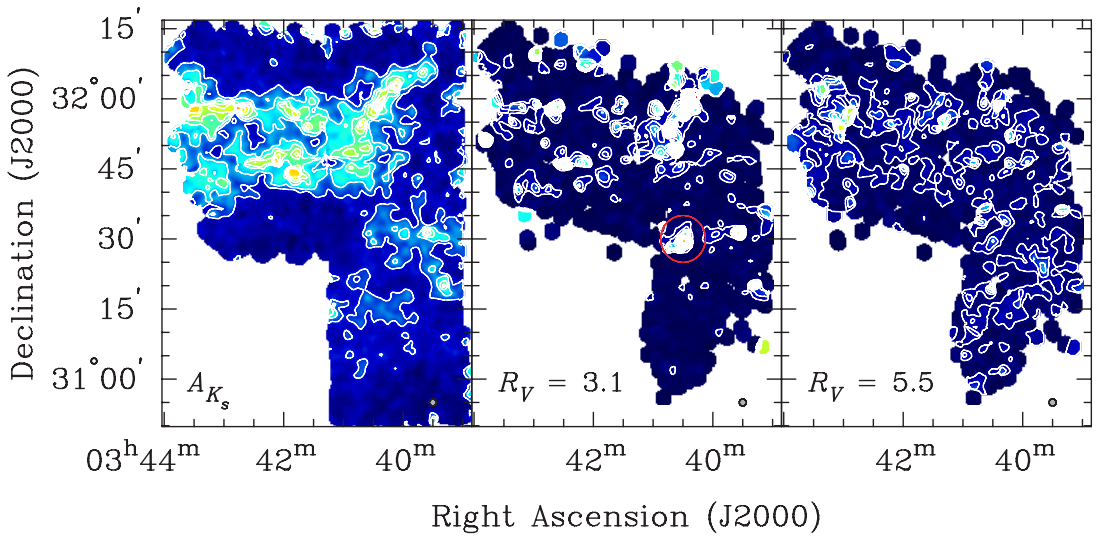

Figure 13. Map of the $A_{K_{s}}$ and $\chi^{2}$ values in Perseus. The left panel shows the extinction map with contours starting at 0.5 mag in steps of 0.25 mag ( $5 \sigma$ ). The middle and right panels show the $\chi^{2}$ maps for the same region made assuming either the WD3.1 (middle) or WD5.5 (right) extinction laws. Contours start at $\chi^{2}=4$ in steps of 4. The region circled in red is discussed in Sections 5.1 and 5.2.3.

(A color version of this figure is available in the online journal.)

unremarkable, suggesting that grain growth may not be causing the increase in $\chi^{2}$. This region will be examined in more detail in Section 5.2.3.

\subsection{The Mid-Infrared Extinction Law}

We have LOS measurements of $A_{K_{s}}$ and the extinction law for every star within our clouds. In the previous section, we turned these measurements into maps of the $\chi^{2}$ deviation from the WD3.1 or WD5.5 extinction laws. Now we will examine the extinction law as a function of wavelength and $A_{K_{s}}$. We started by binning our LOS measurements into four $A_{K_{S}}$ ranges from low to high: $0<A_{K_{s}} \leqslant 0.5,0.5<A_{K_{s}} \leqslant 1,1<A_{K_{s}} \leqslant 2$, and $A_{K_{s}} \geqslant 2$. For each bin, we excluded any negative $C_{\text {ext }}^{\text {obs }}$ values as unphysical and computed the weighted average $C_{\text {ext }}(\lambda)$. If we had used these negative $C_{\mathrm{ext}}^{\mathrm{obs}}$ values, then our extinction law for $0<A_{K_{s}} \leqslant 0.5$ would be $A_{\lambda} / A_{K_{s}} \approx 0.02$ for IRAC2-4 $(4.5-8 \mu \mathrm{m})$, suggesting the dust causes almost no extinction for these wavelengths and far below the predictions of dust models. The impact of negative $C_{\mathrm{ext}}^{\mathrm{obs}}$ values on our other extinction bins is negligible. Our results for our three clouds may be seen in Figures 16-18. The error bars in each bin represent the minimum uncertainty due to systematic errors in measuring the fluxes, propagated through Equation (4).

We plotted three theoretical extinction laws in our figures. The WD $R_{V}=3.1$ and WD $R_{V}=5.5$ models are the same WD3.1 and WD5.5 models, respectively, that we used in computing the $\chi^{2}$. In addition to those two, we plotted a third model, labeled KP v5.0 (K. Pontoppidian et al. 2009, in preparation). This model is one from a grid of models constructed starting from the Weingartner \& Draine (2001) parameterization of the grain size distribution. Icy mantles of water and other volatiles were then added. The specific model we use from this grid is the one with the "best fit" to the c2d mid-IR extinction law and ice features. Several ice absorption features in this model can be seen in the figures, these are due to $\mathrm{H}_{2} \mathrm{O}, \mathrm{CO}_{2}$, or $\mathrm{CO}$. 


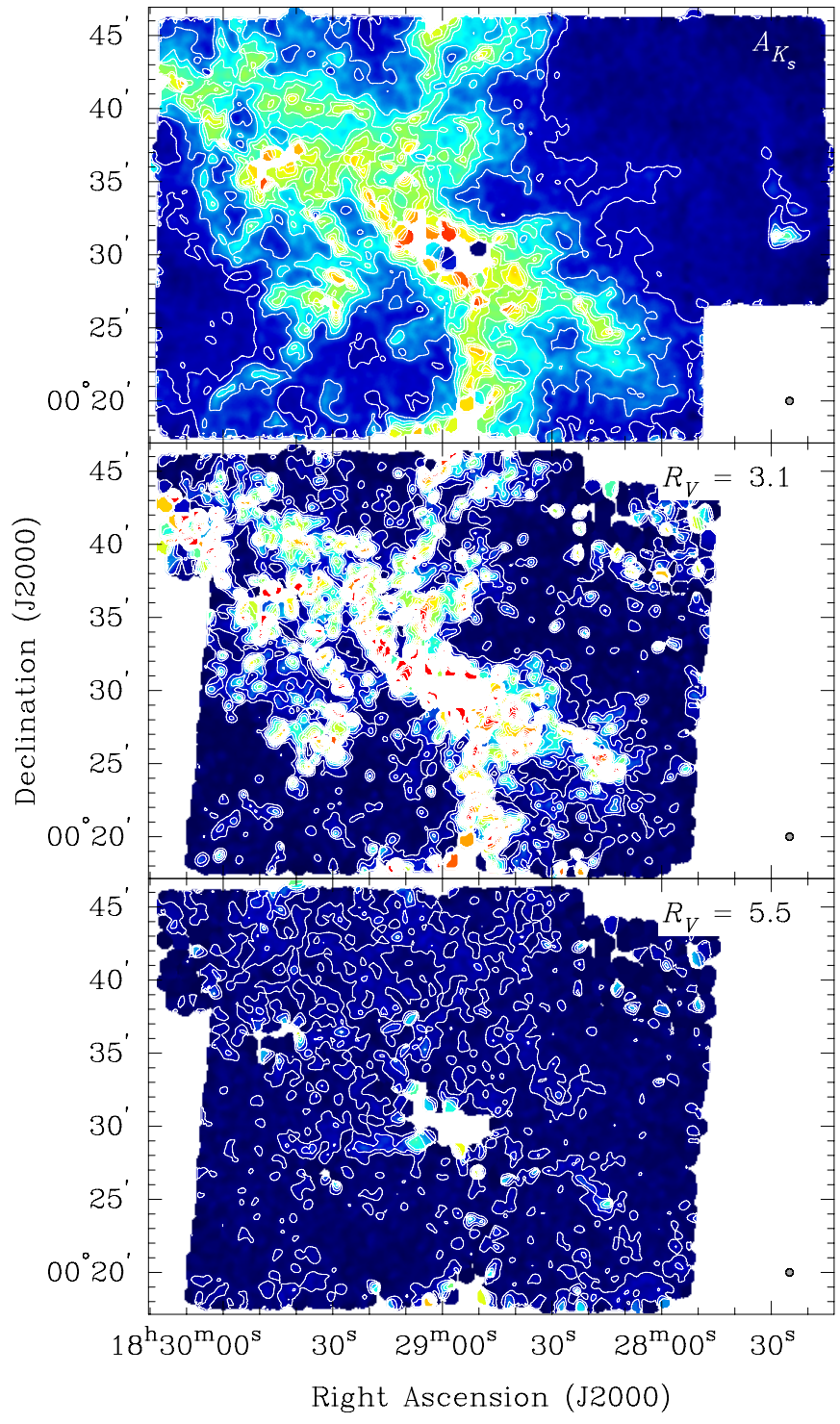

Figure 14. Map of the $A_{K_{s}}$ and $\chi^{2}$ values in Serpens. The top panel shows the extinction map with contours starting at $0.5 \mathrm{mag}$ in steps of $0.25 \mathrm{mag}(5 \sigma)$. The middle and bottom panels are the $\chi^{2}$ maps for the same region made assuming either the WD3.1 (middle) or WD5.5 (bottom) extinction laws. Contours start at $\chi^{2}=4$ in steps of 4 .

(A color version of this figure is available in the online journal.)

\subsubsection{The Extinction Law From 3.6 to $8 \mu \mathrm{m}$}

In the IRAC bands, the observed extinction laws in all three clouds show a similar trend: at low $A_{K_{s}}$, the extinction law is more consistent with the WD3.1 law, while at higher extinctions the observed law gradually flattens to become more consistent with WD5.5. In Table 3 we list our average extinction law, made from all three of our clouds, along with the results from other authors, and also our three dust models.

In the lowest extinction bin, $0<A_{K_{s}} \leqslant 0.5$, the $3.6-8 \mu \mathrm{m}$ extinction law coincides with the law predicted by the WD3.1 dust model, except for $A_{5.8} / A_{K_{s}}=0.28$, which is much higher than the WD3.1 value of 0.17. The data from Perseus and Serpens are the cause of this; both have an anomalously high $A_{5.8} / A_{K_{s}}$ value. If we compute the $\chi^{2}$ statistic and the associated probability, then these data have a $94 \%$ chance of fitting the WD3.1 model $\left(\chi^{2}=0.39\right)$, but only a $22 \%$ chance of fitting the WD5.5 model $\left(\chi^{2}=4.5\right)$.
The extinction law in the $0.5<A_{K_{s}} \leqslant 1$ bin appears to be a transition between the WD3.1 and WD5.5 models. The WD3.1 $\chi^{2}$ is 6.8 , only an $8 \%$ probability of the data matching the model, while the WD5.5 $\chi^{2}$ is 3.8 (29\% probability). For $1<A_{K_{s}} \leqslant 2$, our extinction law is very close to the WD5.5 dust model. It is extremely unlikely that the data fit the WD3.1 model here $\left(\chi^{2}=80,2.5 \times 10^{-15} \%\right.$ probability). Compared to this, the WD5.5 model has $\chi^{2}=1.2$ with a $76 \%$ probability. Finally, for $A_{K_{s}}>2$, our average extinction law is slightly flatter than WD5.5. This is due to the data points from Perseus and Serpens, and is primarily because of the $5.8 \mu \mathrm{m}$ data point. The presence of water ice may explain the $5.8 \mu \mathrm{m}$ relative extinction. The KP v5.0 dust model incorporates the $6.02 \mu \mathrm{m}$ water ice absorption line (Gibb et al. 2004), which is within the IRAC $5.8 \mu \mathrm{m}$ bandpass. The WD3.1 model is even more strongly rejected in this bin $\left(\chi^{2}=303,1.9 \times 10^{-63} \%\right.$ probability $)$, though the WD5.5 model is also a relatively poor fit $\left(\chi^{2}=9.3,2.5 \%\right.$ probability).

As can be seen in Table 3, our 3.6-8 $\mu \mathrm{m}$ extinction law for $A_{K_{s}}>1$ is consistent with the results obtained by other authors. Lutz (1999) measured hydrogen recombination lines toward the Galactic center and computed the extinction law from 2.6 to $19 \mu \mathrm{m}$. Indebetouw et al. (2005) used the IRAC instrument on board Spitzer and measured the extinction law along two lines of sight using data from the Galactic Legacy IR Mid-Plane Survey Extraordinaire (GLIMPSE) Spitzer Legacy Science program (Benjamin et al. 2003). Flaherty et al. (2007) also used Spitzer to measure the extinction law toward five regions. All of these results are in rough agreement; they find a flat extinction law from 3.6 to $8 \mu \mathrm{m}$, consistent with the WD5.5 model.

\subsubsection{The Extinction Law at $24 \mu \mathrm{m}$}

At $24 \mu \mathrm{m}$, the extinction law is generally higher than that predicted by any of the three dust models. This means more extinction exists for a given column density, or, alternatively, our observed $24 \mu \mathrm{m}$ fluxes are fainter than models predict. The exact value of $A_{24} / A_{K_{s}}$ ranges from 0.28 to 1.1 and varies among clouds and extinction bins. The only $A_{24} / A_{K_{s}}$ value which is not higher than any dust model prediction is for $A_{K_{s}}>2$ in Ophiuchus, which is only marginally higher than the predicted value from WD5.5. However, this value is computed from just two stars. This general discrepancy is the reason we excluded the $24 \mu \mathrm{m}$ data when creating our $\chi^{2}$ maps in Section 5.1.

The value of $A_{24} / A_{K_{s}}$ appears to decrease as $A_{K_{s}}$ increases. This behavior is puzzling because if grain growth is occurring in denser regions, as suggested by the $3.6-8 \mu \mathrm{m}$ extinction law, then we would expect regions with a larger column density to have a higher value of $A_{24} / A_{K_{s}}$, not a lower one, since larger grains should have a larger relative extinction. However, we observe the opposite trend.

A likely explanation for this trend is that the average stellar model that we are using is incorrect at $24 \mu \mathrm{m}$. We can obtain an upper limit on the value for the average stellar model flux at $24 \mu \mathrm{m}$. There are 23 stars with $24 \mu \mathrm{m}$ fluxes with $0<A_{K_{s}} \leqslant 0.5$ in all three clouds. If these sources had zero extinction, then the ratio of $24 \mu \mathrm{m}$ to $K_{s}$-band flux for these stars would provide an estimate of the true average stellar flux. However, these stars $d o$ have small amounts of extinction, which will increase the $24 \mu \mathrm{m}$ to $K_{s}$ flux ratio, assuming the true relative extinction at $24 \mu \mathrm{m}$ is less than at $K_{s}$. Thus, our ratio of $24 \mu \mathrm{m}$ to $K_{s}$-band flux is an upper limit on the true average stellar flux. For the 23 low-extinction stars in our clouds, the 
No. 1, 2009

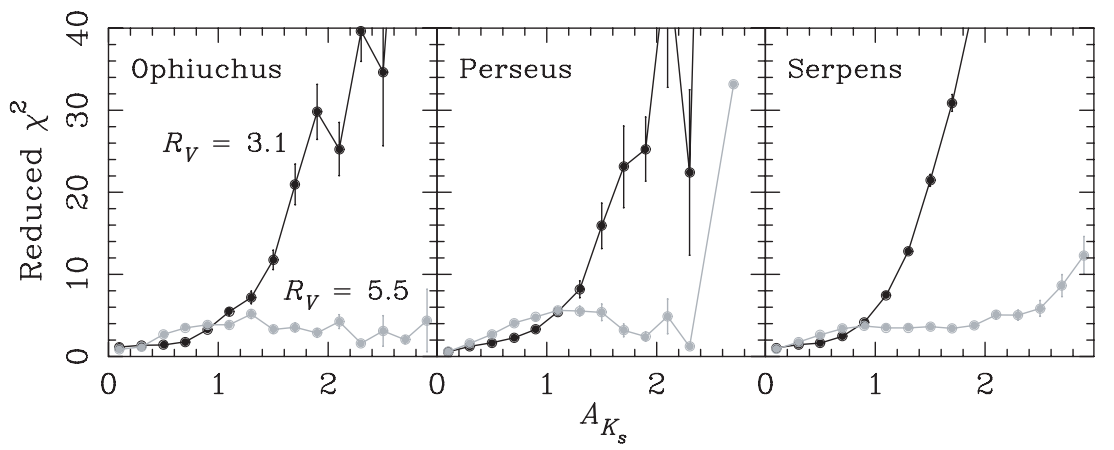

Figure 15. $\chi^{2}$ vs. $A_{K_{s}}$ for the clouds we observed. The WD3.1 $\chi^{2}$ values are black, while the WD5.5 $\chi^{2}$ values are light gray. The error bars shown are the standard deviations of the mean $\chi^{2}$ value in each bin.

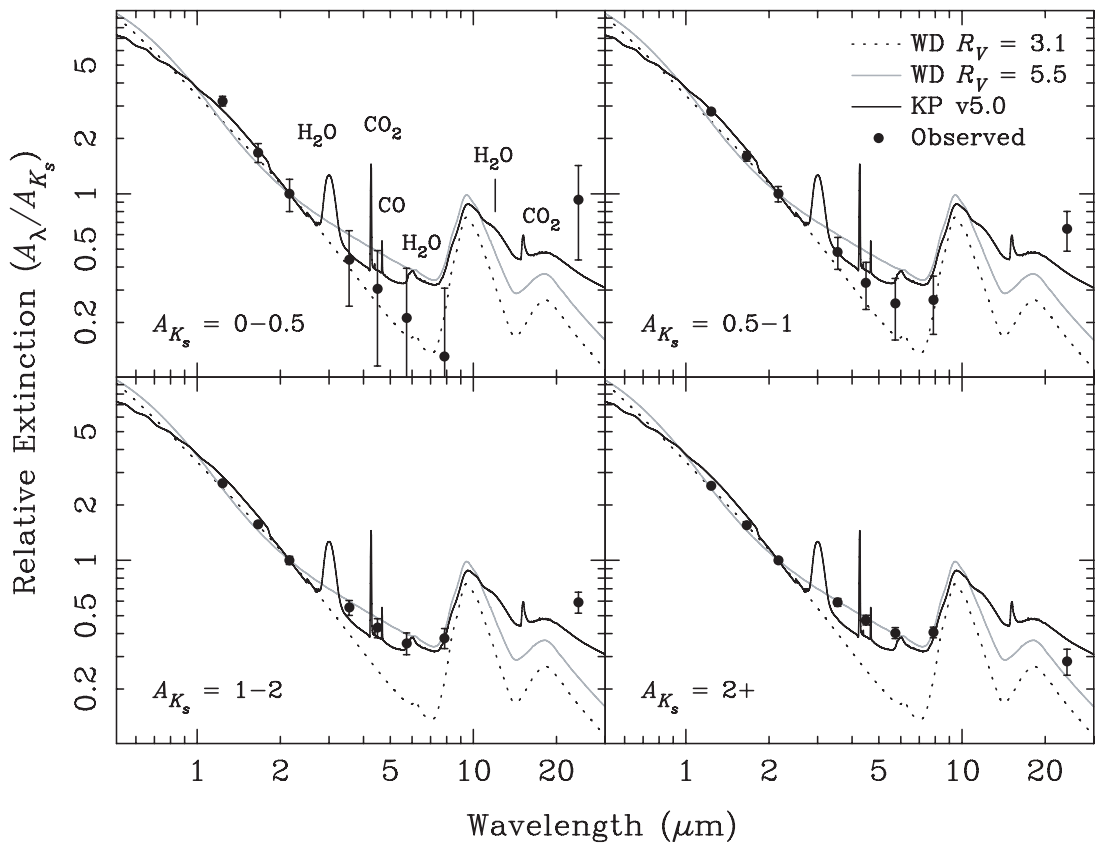

Figure 16. Extinction law in Ophiuchus in four different $A_{K_{s}}$ ranges: $0-0.5,0.5-1,1-2,>2$. The data points are the weighted average observed extinction law computed from all sources within the specified $A_{K_{S}}$ range where the error bars are the minimum uncertainty due to systematic errors in measuring the fluxes. We plotted three different dust models for comparison: Weingartner \& Draine (2001) $R_{V}=3.1$ and 5.5 (dotted, gray lines), and KP v5.0 (K. Pontoppidian et al. 2009 , in preparation) in black. Using Gibb et al. (2004) as a reference, we identified the ice features in KP v5.0.

Table 3

Average Relative Extinction $A_{\lambda} / A_{K_{S}}$

\begin{tabular}{|c|c|c|c|c|c|}
\hline Source & $3.6 \mu \mathrm{m}$ & $4.5 \mu \mathrm{m}$ & $5.8 \mu \mathrm{m}$ & $8 \mu \mathrm{m}$ & $24 \mu \mathrm{m}$ \\
\hline Our data, $0<A_{K_{s}} \leqslant 0.5$ & $0.41 \pm 0.19$ & $0.26 \pm 0.18$ & $0.28 \pm 0.18$ & $0.21 \pm 0.17$ & $1.08 \pm 0.32$ \\
\hline Our data, $0.5<A_{K_{s}} \leqslant 1$ & $0.49 \pm 0.10$ & $0.35 \pm 0.10$ & $0.35 \pm 0.10$ & $0.35 \pm 0.10$ & $0.75 \pm 0.14$ \\
\hline Our data, $1<A_{K_{s}} \leqslant 2$ & $0.60 \pm 0.05$ & $0.46 \pm 0.05$ & $0.44 \pm 0.05$ & $0.43 \pm 0.05$ & $0.61 \pm 0.08$ \\
\hline Our data, $A_{K_{s}} \geqslant 2$ & $0.64 \pm 0.03$ & $0.53 \pm 0.03$ & $0.46 \pm 0.03$ & $0.45 \pm 0.03$ & $0.34 \pm 0.13$ \\
\hline \multicolumn{6}{|c|}{ Other Authors } \\
\hline Flaherty et al. (2007) & $0.632 \pm 0.004$ & $0.54 \pm 0.01$ & $0.50 \pm 0.02$ & $0.50 \pm 0.01$ & $0.46 \pm 0.04$ \\
\hline Indebetouw et al. (2005) & $0.56 \pm 0.06$ & $0.43 \pm 0.08$ & $0.43 \pm 0.10$ & $0.43 \pm 0.10$ & $\cdots$ \\
\hline Lutz (1999)a & $0.53 \pm 0.03$ & $0.50 \pm 0.08$ & $0.49 \pm 0.06$ & $0.42 \pm 0.06$ & $\cdots$ \\
\hline \multicolumn{6}{|c|}{ Dust Models ${ }^{\mathrm{b}}$} \\
\hline Weingartner \& Draine (2001), $R_{V}=3.1$ & 0.40 & 0.25 & 0.17 & 0.22 & 0.17 \\
\hline Weingartner \& Draine (2001), $R_{V}=5.5$ & 0.60 & 0.49 & 0.40 & 0.41 & 0.24 \\
\hline $\mathrm{KP}, \mathrm{v} 5.0$ & 0.48 & 0.38 & 0.34 & 0.38 & 0.38 \\
\hline
\end{tabular}

Notes.

${ }^{a}$ The extinction for the closest Infrared Space Observatory wavelength to each Spitzer band is listed: 3.7, 4.4, 5.9, and $7.5 \mu \mathrm{m}$.

${ }^{\mathrm{b}}$ Extinctions are computed at the central wavelength in each band. 


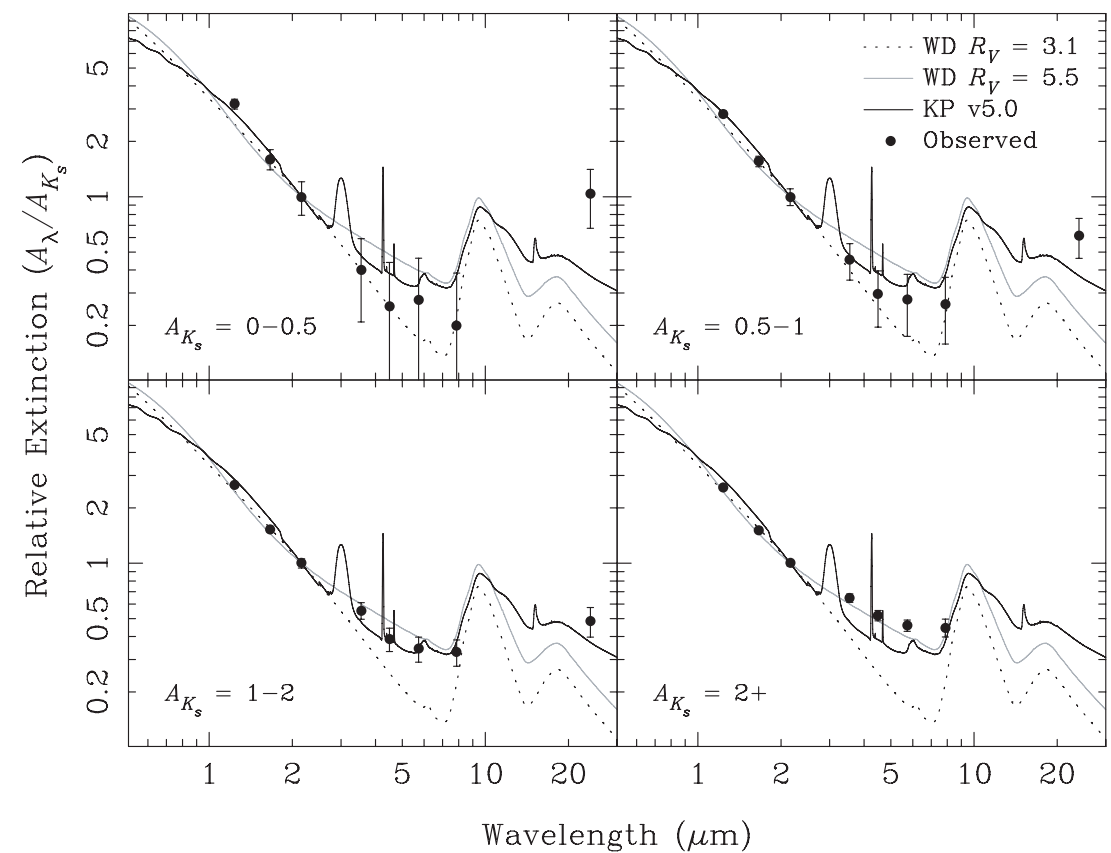

Figure 17. Extinction law in Perseus in four different $A_{K_{s}}$ ranges: $0-0.5,0.5-1,1-2,>2$. See Figure 16 for a description of the data points, curves, and error bars.

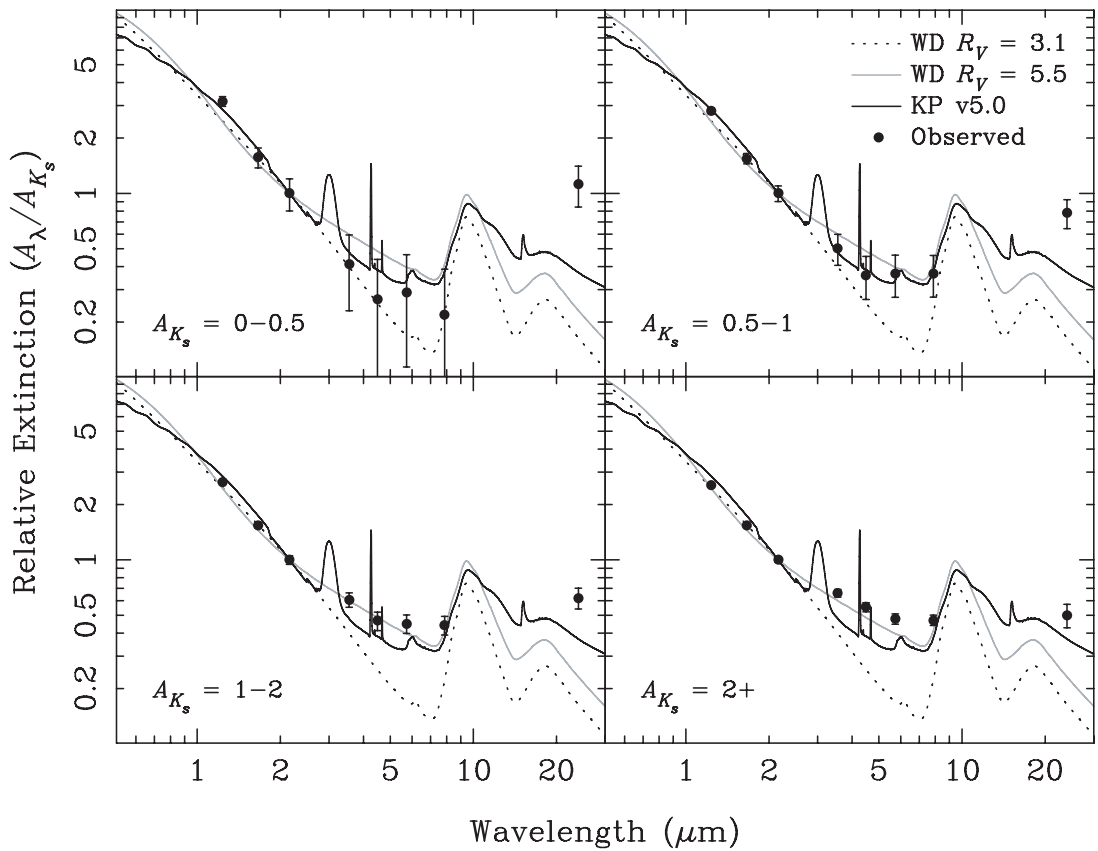

Figure 18. Extinction law in Serpens in four different $A_{K_{s}}$ ranges: $0-0.5,0.5-1,1-2,>2$. See Figure 16 for a description of the data points, curves, and error bars.

Table 4

Average Stellar Model for Each Cloud

\begin{tabular}{lcccccccc}
\hline \hline Region & $\begin{array}{c}J \\
(\mathrm{mJy})\end{array}$ & $\begin{array}{c}H \\
(\mathrm{mJy})\end{array}$ & $\begin{array}{c}K_{s} \\
(\mathrm{mJy})\end{array}$ & $\begin{array}{c}\text { IRAC1 } \\
(\mathrm{mJy})\end{array}$ & $\begin{array}{c}\text { IRAC2 } \\
(\mathrm{mJy})\end{array}$ & $\begin{array}{c}\text { IRAC3 } \\
(\mathrm{mJy})\end{array}$ & $\begin{array}{c}\text { IRAC4 } \\
(\mathrm{mJy})\end{array}$ & $\begin{array}{c}\text { MIPS1 } \\
(\mathrm{mJy})\end{array}$ \\
\hline Ophiuchus & $1.374 \pm 0.291$ & $1.335 \pm 0.095$ & 1.0 & $0.448 \pm 0.018$ & $0.267 \pm 0.011$ & $0.178 \pm 0.008$ & $0.103 \pm 0.006$ & $0.014 \pm 0.001$ \\
Perseus & $1.238 \pm 0.276$ & $1.284 \pm 0.099$ & 1.0 & $0.457 \pm 0.023$ & $0.269 \pm 0.011$ & $0.180 \pm 0.013$ & $0.101 \pm 0.008$ & $0.014 \pm 0.000$ \\
Serpens & $1.560 \pm 0.232$ & $1.393 \pm 0.061$ & 1.0 & $0.443 \pm 0.013$ & $0.261 \pm 0.016$ & $0.177 \pm 0.005$ & $0.106 \pm 0.005$ & $0.014 \pm 0.000$ \\
Average & $1.412 \pm 0.152$ & $1.357 \pm 0.046$ & 1.0 & $0.447 \pm 0.010$ & $0.267 \pm 0.007$ & $0.178 \pm 0.004$ & $0.104 \pm 0.003$ & $0.014 \pm 0.001$ \\
\hline
\end{tabular}

flux ratio is $0.014 \pm 0.002$, the same value listed in Table 4 . Since the average extinction for these stars is $0.3 A_{K_{s}}$, the true value will be less than this. As a test, we took our combined catalogs for all three clouds and used a smaller average model flux of $0.012 \mathrm{mJy}$ at $24 \mu \mathrm{m}$. In our four extinction bins, our relative extinction becomes (from low to high $A_{K_{s}}$ ): $0.65 \pm$ $0.31,0.56 \pm 0.14,0.52 \pm 0.08$, and $0.28 \pm 0.12$. Using a lower value for the $24 \mu \mathrm{m}$ stellar flux almost completely eliminates 


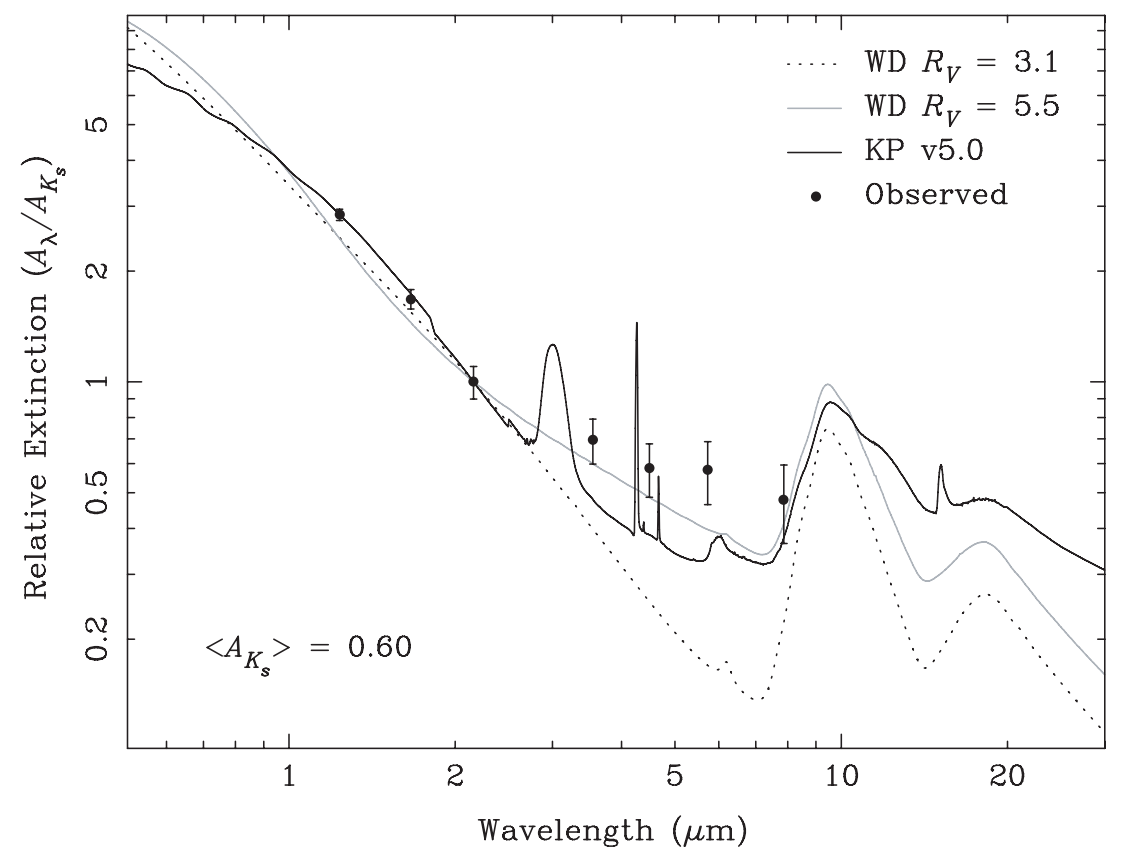

Figure 19. Average extinction law obtained for the region in Perseus with anomalously high $\chi^{2}$ in the $R_{V}=3.1$ map (see Sections 5.1 and 5.2.3). The coordinates of this region are $\left(\sim 3^{\mathrm{h}} 40^{\mathrm{m}} \cdot 5+31^{\circ} 30^{\prime}\right)$. See Figure 16 for a description of the data points, curves, and error bars.

the trend of $A_{24} / A_{K_{s}}$ decreasing as $A_{K_{s}}$ increases. The only extinction bin which shows a significant drop in the relative extinction is for $A_{K_{s}}>2$, but there are only three $24 \mu \mathrm{m}$ stars in this bin. A larger sample of stars is needed to confirm this low value.

Other authors also found $A_{24} / A_{K_{s}}$ to be higher than predicted by dust models. Their values are in rough agreement with ours, assuming we use the lower value for the average stellar flux proposed above. Flaherty et al. (2007) were able to measure $A_{24} / A_{K_{s}}$ for two of their five regions and found $A_{24} / A_{K_{s}}=$ $0.44 \pm 0.02$ and $0.52 \pm 0.03$ for Serpens and NGC2068/71, respectively. However, they caution that their sample size is small. Even though Lutz (1999) did not measure the extinction longward of $19 \mu \mathrm{m}$, if their flat extinction law was projected out to $24 \mu \mathrm{m}$, it would also be $\sim 0.5$.

It is clear that the extinction law at $24 \mu \mathrm{m}$ is not well understood. With some assumptions about the average stellar flux, our findings are consistent with those of Flaherty et al. (2007). Both their and our results suggest $A_{24} / A_{K_{s}} \sim 0.5$, or 2-3 times larger than the value predicted by current dust models. New dust models will need to incorporate additional extinction at $24 \mu \mathrm{m}$ to reproduce these observations. Furthermore, both the Flaherty et al. and our results are from relatively small samples. A larger study is needed before we can answer questions about variations in the $24 \mu \mathrm{m}$ extinction law with column density or LOS.

\subsubsection{Perseus $\chi^{2}$ Map}

In this section we will address the "anomalous" region in the Perseus $R_{V}=3.1 \chi^{2}$ map. This region is circled in red in Figure 13. Fifty-three stars are within the region defined by the $\chi^{2}=4.0$ contour. We plotted the extinction law derived from these 53 stars in Figure 19 and found the extinction law for these data is significantly higher than the WD5.5 model with the largest deviation occurring at $5.8 \mu \mathrm{m}$. The water ice feature at $6.02 \mu \mathrm{m}$ may explain the discrepancy, however ices are seen toward dense cores and the average extinction for this region is a relatively modest $A_{K_{s}}=0.60$ (Gibb et al. 2004; Knez et al. 2005). Another possibility is that the sources in this region may not really be stars, but a search of the NASA/IPAC Extragalactic Database and Simbad within $2^{\prime}$ of $3^{\mathrm{h}} 40^{\mathrm{m}} \cdot 5+31^{\circ} 30^{\prime}$ yielded no matches. A final possibility is that the NIR extinction law is different in this region compared to our assumed WD5.5. We will see how this impacts our mid-IR extinction law in the following section.

\subsection{Changes in the Near-Infrared Extinction Law}

As we mentioned in the introduction, the NIR extinction law can be fitted by a power law, $A_{\lambda} \propto \lambda^{-\beta}$, with $\beta=$ 1.6-1.8 (Draine 2003, and references therein). For this paper we assumed the WD5.5 model, with $\beta \approx 1.6$, is correct in the NIR. This assumption allows us to directly compare our results with those of other authors, who have made the same assumption. Furthermore, our data agree with the $\beta=1.6$ reddening vector shown in Figure 5. However, if the NIR extinction law is actually steeper in some regions this will introduce another source of error into our measurements of the mid-IR extinction law. To measure the effect of choosing a different value for $\beta$, we modified the WD5.5 law to have $\beta=1.8$ in the NIR, then re-classified the stars, computed $A_{K_{s}}$, and derived the mid-IR extinction law. Our values of $A_{\lambda} / A_{K_{s}}$ are decreased by using $\beta=1.8$ as opposed to WD5.5. The decrease is $\sim 15 \%$ for IRAC 1 and IRAC2, $\sim 25 \%$ for IRAC 3 and IRAC4, and $\sim 15 \%$ for MIPS1. The extinction law in Figures 16-18 still flattens as $A_{K_{s}}$ increases, but does so more gradually. A different value of $\beta$ may partially explain the "anomalous" region in Perseus. Using the $\beta=1.8$ law for this region, we found that the derived extinction law in the mid-IR is decreased to the point that it is consistent with WD5.5. However, the extinction law is still flatter than what is observed elsewhere in Perseus at the same $A_{K_{s}}$.

Based on this analysis, we expect our assumption of the WD5.5 extinction law in the NIR may introduce an error of up to $15 \%$ in IRAC1, IRAC2, and MIPS1, and 25\% in IRAC3 
and IRAC4. However, we have no way of measuring possible variations in the NIR extinction law along different lines of sight. Therefore, while it is important to keep this in mind as a possible source of error, we have not attempted to incorporate it into the error bars shown in our figures.

\section{CONCLUSIONS}

In this paper we took deep $J H K_{s}$ observations of regions within three molecular clouds: Ophiuchus, Perseus, and Serpens. These were combined with Spitzer c2d data to create band-merged catalogs in eight wave bands from 1.2 to $24 \mu \mathrm{m}$. Our final catalogs contain 2365 stars in Ophiuchus, 11,280 in Perseus, and 49,485 in Serpens. From these catalogs we created uniform maps of the extinction and $\chi^{2}$. We also computed the average extinction law in four extinction bins: $0<A_{K_{s}} \leqslant 0.5$, $0.5<A_{K_{s}} \leqslant 1,1<A_{K_{s}} \leqslant 2$, and $A_{K_{s}}>2$.

Our results in the IRAC bands, 3.6-8 $\mu \mathrm{m}$, show that grain growth occurs in the dense regions of our clouds. The $\chi^{2}$ maps show the deviations from two dust models: Weingartner \& Draine (2001) with $R_{V}=3.1$ and $R_{V}=5.5$ (abbreviated as WD3.1 and WD5.5). There is a strong correlation between highextinction and high-WD3.1 $\chi^{2}$, but not with WD5.5 $\chi^{2}$. This suggests denser regions are more consistent with the WD5.5 extinction model, which has grain sizes up to 10 times larger than those in the WD3.1 dust model. The same behavior is seen in the average extinction law. For $0<A_{K_{s}} \leqslant 0.5$, it is most consistent with WD3.1 (94\% probability of the data fitting this model). As $A_{K_{s}}$ increases, the extinction law gradually flattens and becomes more consistent with WD5.5 for $1<A_{K_{s}} \leqslant 2$, with a $76 \%$ probability. For $A_{K_{s}}>2$, the WD5.5 model is not as good a fit to the data, primarily due to the $A_{5.8} / A_{K_{s}}$ relative extinction, which is higher than the model. This may indicate the presence of water ice in the densest regions.

Not all areas in our clouds can be explained using grain growth. Our $\chi^{2}$ map of Perseus reveals a region that has only a modest average extinction of $A_{K_{s}}=0.60$, yet has a peak WD3.1 $\chi^{2}$ of 39. The extinction law in this region is much flatter than WD5.5. Because of the low average extinction here, it does not seem likely that grain growth is altering the extinction law, nor that ices have formed on the dust grains. It is possible the background sources in this region are not really stars, or that the NIR extinction law is steeper in this region. More work is needed to understand the physical cause of the extinction law in this region.

Unlike in the IRAC bands, the $24 \mu \mathrm{m}$ extinction law is $2-4$ times higher than the current dust models predict. There is also a negative correlation between $A_{K_{s}}$ and the value of $A_{24} / A_{K_{s}}$. Both of these results maybe partially mitigated by considering the small numbers of stars detected at $24 \mu \mathrm{m}$ or slight changes to our average stellar model flux. However, even with the most conservative assumptions, our average value for $A_{24} / A_{K_{s}}$ is still $\sim 2$ times larger than that predicted by dust-grain models. This result agrees with the measured values from Flaherty et al. (2007). Future dust models will need to reproduce the relatively large value of the $24 \mu \mathrm{m}$ extinction.

We thank the anonymous referee whose useful comments improved this paper. Support for this work, utilizing data from the "Cores to Disks" Spitzer Legacy Science Program (Evans et al. 2003), was provided by NASA through contracts 1224608 , 1230782, and 1230779 issued by the Jet Propulsion Laboratory, California Institute of Technology, under NASA contract 1407.
Additional support for N.L.C. was provided by NASA through JPL contracts 1264793 and 1264492. L.G.M. was supported by NASA Origins Grant NAG510611. This research has made use of PyRAF, a product of the Space Telescope Science Institute, which is operated by AURA for NASA. We also used SIMBAD and the NASA/IPAC Extragalactic Database (NED). The SIMBAD database is operated at CDS, Strasbourg, France. NED is operated by the Jet Propulsion Laboratory, California Institute of Technology, under contract with the National Aeronautics and Space Administration.

\section{APPENDIX}

\section{AVERAGE STELLAR MODELS}

To compute the expected stellar distribution for each cloud, we used the galaxy model from Jarrett (1992) and Jarrett et al. (1994). Given the Galactic coordinates, distance, and average extinction for a cloud, this model produces source counts broken down by spectral type. These source counts provide a rudimentary weighting function that could be used in computing the average stellar model. However, a weighting function from the raw source counts would ignore the reality that not all spectral types are equally observable at each wavelength. Our next step was to correct for the detection limits of our actual observations. The galaxy model lists the extincted $K_{s}$ magnitude for each star and its extinction, $A_{V}$. We used this information, the Weingartner and Draine $R_{V}=3.1$ extinction law, and the stellar templates from "Star-Pet" to compute the magnitude of each star at every observed wave band from $J-24 \mu \mathrm{m}$. We used WD3.1 since this was the law used by the galaxy model when computing the extinctions. We arbitrarily discarded any detection falling below the $5 \sigma$ cutoff for the given wave band (Table 2). Using the normalized, final, corrected source counts at each wave band as a weighting function, we then computed the average stellar template for each region and an overall average stellar model. Our results are shown in Table 4 . We repeated the above procedure using the Weingartner and Draine $R_{V}=5.5$ extinction law. The average fluxes using this law never vary by more than $0.001 \mathrm{mJy}$ from those listed in Table 4.

\section{REFERENCES}

Benjamin, R. A., et al. 2003, PASP, 115, 953

Bohlin, R. C., Savage, B. D., \& Drake, J. F. 1978, ApJ, 224, 132

de Geus, E. J., de Zeeuw, P. T., \& Lub, J. 1989, A\&A, 216, 44

Draine, B. T. 2003, ARA\&A, 41, 241

Elston, R. 1998, in Proc. SPIE 3354, Infrared Astronomical Instrumentation, ed. A. M. Fowler, 404-413

Enoch, M. L., et al. 2006, ApJ, 638, 293

Evans, N. J., II, et al. 2003, PASP, 115, 965

Flaherty, K. M., Pipher, J. L., Megeath, S. T., Winston, E. M., Gutermuth, R. A., Muzerolle, J., Allen, L. E., \& Fazio, G. G. 2007, ApJ, 663, 1069

Foster, J. B., Román-Zúñiga, C., Goodman, A. A., Lada, E., \& Alves, J. 2008, ApJ, 674, 831

Gibb, E. L., Whittet, D. C. B., Boogert, A. C. A., \& Tielens, A. G. G. M. 2004, ApJS, 151, 35

Harvey, P. M., et al. 2006, ApJ, 644, 307

Harvey, P., Merín, B., Huard, T. L., Rebull, L. M., Chapman, N., Evans, N. J., II, \& Myers, P. C. 2007a, ApJ, 663, 1149

Harvey, P. M., et al. 2007b, ApJ, 663, 1139

Indebetouw, R., et al. 2005, ApJ, 619, 931

Jarrett, T. H. 1992, PhD thesis, AA, Univ. of Massachusetts, Amherst

Jarrett, T. H., Dickman, R. L., \& Herbst, W. 1994, ApJ, 424, 852

\footnotetext{
http://ssc.spitzer.caltech.edu/tools/starpet
} 
Jørgensen, J. K., et al. 2006, ApJ, 645, 1246

Knez, C., et al. 2005, ApJ, 635, L145

Koornneef, J. 1983, A\&A, 128, 84

Lombardi, M., \& Alves, J. 2001, A\&A, 377, 1023

Lutz, D. 1999, The Universe as Seen by ISO, ed. P. Cox \& M. F. Kessler, ESA SP-427, 623
Padgett, D. L., et al. 2008, ApJ, 672, 1013

Rebull, L. M., et al. 2007, ApJS, 171, 447

Rieke, G. H., \& Lebofsky, M. J. 1985, ApJ, 288, 618

Straizys, V., Cernis, K., \& Bartasiute, S. 1996, Baltic Astron., 5, 125

Surace, J. A., et al. 2004, VizieR Online Data Catalog II/255

Weingartner, J. C., \& Draine, B. T. 2001, ApJ, 548, 296 